\title{
Structure-informed microbial population genetics elucidate selective pressures that shape protein evolution
}

\author{
Evan Kiefl ${ }^{1,2,{ }^{*},}$ Ozcan C. Esen ${ }^{1}$, Samuel E. Miller ${ }^{1}$, Kourtney L. Kroll², Amy D. Willis ${ }^{3}$, \\ Michael S. Rappé ${ }^{4}$ Tao $\operatorname{Pan}^{5}$, A. Murat Eren ${ }^{1,6,7,8,{ }^{*}}$
}

\begin{abstract}
${ }^{1}$ Department of Medicine, University of Chicago, Chicago, IL, USA; ${ }^{2}$ Graduate Program in Biophysical Sciences, University of Chicago, Chicago, IL, USA; ${ }^{3}$ Department of Biostatistics, University of Washington, Seattle, WA, USA; ${ }^{4}$ Hawai'i Institute of Marine Biology, University of Hawai'i at Mānoa, Kāne'ohe, Hawai'i, USA; ${ }^{5}$ Department of Biochemistry \& Molecular Biology, University of Chicago, Chicago, IL, USA; ${ }^{6}$ Josephine Bay Paul Center for Comparative Molecular Biology and Evolution, Marine Biological Laboratory, Woods Hole, MA 02543, USA; ${ }^{7}$ Helmholtz Institute for Functional Marine Biodiversity, Oldenburg, Germany; ${ }^{8}$ Institute for Chemistry and Biology of the Marine Environment, University of Oldenburg, Oldenbug, Germany.
\end{abstract}

*Correspondence: ekief|@uchicago.edu and meren@uchicago.edu

\section{Running Title}

Structure-informed microbial population genetics

\section{Keywords}

evolution, metagenomics, SAR11, AlphaFold, anvi'o structure 


\section{Abstract}

1 Comprehensive sampling of natural genetic diversity with metagenomics enables highly resolved

2 insights into the interplay between ecology and evolution. However, intra-population genomic

3 variation represents the outcome of both stochastic and selective forces, making it difficult to

4 identify whether variants are maintained by adaptive, neutral, or purifying processes. This is partly

5 due to the reliance on gene sequences to interpret variants, which disregards the physical

6 properties of three-dimensional gene products that define the functional landscape on which

7 selection acts. Here we describe an approach to analyze genetic variation in the context of

8 predicted protein structures, and apply it to study a marine microbial population within the SAR11

9 subclade 1a.3.V, which dominates low-latitude surface oceans. Our analyses reveal a tight

10 association between the patterns of nonsynonymous polymorphism, selective pressures, and

11 structural properties of proteins such as per-site relative solvent accessibility and distance to

12 ligands, which explain up to $59 \%$ of genetic variance in some genes. In glutamine synthetase, a

13 central gene in nitrogen metabolism, we observe decreased occurrence of nonsynonymous

14 variants from ligand binding sites as a function of nitrate concentrations in the environment,

15 revealing genetic targets of distinct evolutionary pressures maintained by nutrient availability. Our

16 data also reveals that rare codons are purified from ligand binding sites when genes are under

17 high selection, demonstrating the utility of structure-aware analyses to study the variants that

18 likely impact translational processes. Overall, our work yields insights into the governing principles

19 of evolution that shape the genetic diversity landscape within a globally abundant population, and

20 makes available a software framework for structure-aware investigations of microbial population

21 genetics. 


\section{Significance}

22 Increasing availability of metagenomes offers new opportunities to study evolution, but the equal

23 treatment of all variants limits insights into drivers of sequence diversity. By capitalizing on recent

24 advances in protein structure prediction capabilities, our study examines subtle evolutionary

25 dynamics of a microbial population that dominates surface oceans through the lens of structural

26 biology. We demonstrate the utility of structure-informed metrics to understand the distribution of

27 nonsynonymous polymorphism, establish insights into the impact of changing nutrient availability

28 on protein evolution, and show that even synonymous variants are scrutinized strictly to maximize

29 translational efficiency when selection is high. Overall, our work illustrates new opportunities for

30 discovery at the intersection between metagenomics and structural bioinformatics, and offers an

31 interactive and scalable software platform to visualize and analyze genetic variants in the context

32 of predicted protein structures and ligand-binding sites. 


\section{Introduction}

33 Genetic diversity within populations emerges from and is shaped by a combination of stochastic

34 and selective pressures, which often lead to phenotypic differences between closely related

35 individuals, sometimes within a few generations (Burke et al. 2010; Lenski et al. 1991). Surveys

36 of microbial communities within natural habitats through phylogenetic marker genes (Olsen et al.

37 1986; Acinas et al. 2004; Sogin et al. 2006) and metagenomics (Simmons and DiBartolo et al.

38 2008; Allen et al. 2007) have revealed a tremendous amount of genetic variation within

39 environmental populations (T. P. Curtis and Sloan 2005; Thomas P. Curtis et al. 2006), and an

40 ever-increasing number of available genomes and metagenomes have provided insight into the

41 selective pressures that shape such variation. However, the overwhelming complexity and

42 dynamicity of these selective pressures, which occur even in the simplest environments (Good et

43 al. 2017), has hindered our ability to determine which variants are under the influence of which

44 pressures (Ochman 2003; Mes 2008).

45 Inferring selective pressures through the isolation of microbial strains and comparative genomics

46 has been widely successful. More recently, metagenome-assembled genomes (L.-X. Chen et al.

47 2020) and single-amplified genomes (Woyke, Doud, and Schulz 2017) have dramatically

48 increased the number (Almeida et al. 2021; Pachiadaki et al. 2019; Paoli et al. 2021) and diversity

49 (Hug et al. 2016) of microbial clades represented in genomic databases, offering an even larger

50 sampling of environmental microbes to study the emergence and maintenance of genetic variation

51 (Garud and Pollard 2020). Nevertheless, genomes represent static snapshots of individual

52 members of often complex environmental populations, and thus, working with genomic

53 sequences alone substantially undersamples genetic variability in natural habitats and its

54 associations with environmental and ecological forces (Van Rossum et al. 2020). This

55 shortcoming is partially addressed by shotgun metagenomics (Quince et al. 2017) and 
metagenomic read recruitment, where environmental sequences that are aligned to a reference can be studied to identify genetic variants at the resolution of single nucleotides (Whitaker and Banfield 2006; Denef 2019). In particular, using genomes to recruit reads from metagenomes enables a comprehensive sampling of all genetic variants within environmental populations (Simmons and DiBartolo et al. 2008). Due to the immensity of sequencing data generated by metagenomic studies, even subtle genetic variation in natural populations is now resolvable, making it possible to explicitly correlate patterns of genomic variation with temporal or spatial environmental variables to elucidate the interplay between ecology and evolution (Schloissnig et al. 2013; Bendall et al. 2016; Anderson et al. 2017; Delmont et al. 2019; Garud et al. 2019; Zhao et al. 2019; Shenhav and Zeevi 2020; Olm et al. 2021; Conwill et al. 2022). Although quantification and analysis of sequence variants derived from metagenomic data has improved dramatically, inferring the functional impact of individual nucleotides remains a fundamental challenge in part due to the sole reliance on DNA sequences, which do not represent physical properties of proteins they encode, and thus disguise the functional impact of individual mutations.

Given the intermediary role that structure plays within the 'sequence-structure-function paradigm' (Anfinsen 1973), including protein structures as a dimension of analysis is commonplace in

72 studies of protein evolution (Siltberg-Liberles, Grahnen, and Liberles 2011; Harms and Thornton

73 2013; Sikosek and Chan 2014), and it is appreciated that the accuracy of evolutionary models

74 improves with combined analyses of protein structures and the evolution of underlying sequences

75 (Wilke 2012). In contrast, the state-of-the-art approaches that quantify genetic variants in environmental microbial populations typically treat genes as strings of nucleotides (Schloissnig et

77 al. 2013; Eren et al. 2015; Nayfach et al. 2016; Costea et al. 2017; Olm et al. 2021). While this

78 strategy enables rapid surveys of population dynamics through single-nucleotide variants, it 79 disregards the physical properties of three-dimensional gene products that selection acts upon, 80 and thus misses a critical intermediate to understand the relationship between selection and 
81 fitness (Golding and Dean 1998; K. Chen and Arnold 1993). The importance of mapping

82 sequence variants on predicted protein structures to identify genetic determinants of phenotypic

83 variation has been noted more than two decades ago (Sunyaev, Lathe, and Bork 2001), yet the

84 limited availability of protein structures has historically rendered protein structure-informed

85 microbial population genetics impractical. Given dramatic advances in both solving and predicting

86 protein structures in recent years (Kuhlman and Bradley 2019), most notably deep learning

87 approaches such as AlphaFold (Jumper et al. 2021) that offer highly accurate protein structure

88 predictions, this constraint is likely a problem of the past. Altogether, open questions in microbial

89 ecology and evolution, advances in computation, and increased availability of data are

90 culminating in a research landscape that is ripe for new software solutions that integrate protein

91 structures with 'omics data in order to observe and interpret evolutionary processes that shape

92 sequence variation in natural populations.

93 Here we develop an interactive and scalable software solution for the analysis and interactive

94 visualization of metagenomic sequence variants in the context of predicted protein structures and

95 ligand binding sites as a new module in anvi'o, an open-source, community-led multi-omics

96 platform (https://anvio.org). By importing AlphaFold-predicted protein structures into anvi'o

97 structure, we (1) demonstrate the shortcomings of purely sequence-based approaches to interpret

98 patterns of polymorphism observed within complex microbial populations, (2) propose two

99 structural features to interpret genetic variation, RSA and DTL, (3) illustrate that nonsynonymous

100 polymorphism is more likely to encroach upon active sites when selection is low, but is purged

101 from active sites when selection is high, and (4) provide evidence that common codons are more

102 translationally robust than their rare synonymous counterparts, which appear within

103 structurally/functionally noncritical sites when selection is low. 


\section{Results and Discussion}

104 To investigate selective pressures that drive protein evolution within microorganisms inhabiting 105 complex naturally occurring environments, we chose a single microbial taxon and a set of 106 metagenomes that match to its niche boundaries: SAR11 (Candidatus Pelagibacter ubique), a 107 microbial clade of free-living heterotrophic alphaproteobacteria that dominates surface ocean 108 waters (Morris et al. 2002), and Tara Oceans Project metagenomes (Sunagawa et al. 2015), a 109 massive collection of deeply sequenced marine samples from oceans and seas across the globe.

110 SAR11 is divided into multiple subclades with distinct ecology (Giovannoni 2017). Thus, we

111 further narrowed our focus to HIMB83, a single SAR11 strain genome that is $1.4 \mathrm{Mbp}$ in length.

112 HIMB83 is a member of the environmental SAR11 lineage 1a.3.V, one of the most abundant

113 (Nayfach et al. 2016) and most diverse (Delmont \& Kiefl et al. 2019) microbial lineages in marine

114 systems, which recruits as much as $1.5 \%$ of all metagenomic short reads in surface ocean 115 metagenomes (Delmont \& Kiefl et al. 2019).

116 To quantify the genetic variability of $1 \mathrm{a} .3 . \mathrm{V}$, we used HIMB83 as a reference genome of the 117 subclade, and competitively recruited short reads (see Methods) from 93 low-latitude surface 118 ocean metagenomes (Table S1), resulting in 390 million reads that were $94.5 \%$ identical to 119 HIMB83 on average (Figure S1). As an individual member of a diverse subclade, HIMB83 120 possesses a genomic context that is insufficient for resolving the extent of genetic diversity within 121 1a.3.V. Regardless, HIMB83 possesses the 'core' gene set of 1a.3.V, and so reads recruited by 122 these genes represent the diversity of the 1a.3.V core genome. Of the 1,470 genes in HIMB83, 123 we restricted our analysis to 799 genes that we determined to form the 1a.3.V core genes, and 12474 metagenomes in which the average coverage of HIMB83 exceeded 50X (see Methods). The 125 reads recruited to the 1a.3.V core represent a dense sampling of the diversity within this 126 environmental lineage that far exceeds the evolutionary resolution and volume of sequence data 
127 achievable through comparisons of cultured SAR11 genomes alone (Figure S1). As a result,

128 these data provide a unique opportunity to zoom in and track how genomic variation in one of the

129 most abundant microbial populations on Earth shifts in response to ecological parameters

130 throughout the global ocean (Figure S2).

\section{Polymorphism rates reveal intense purification of nonsynonymous}

\section{mutants}

131 To quantify genomic variation in 1a.3.V, in each sample we identified codon positions of HIMB83

132 where aligned metagenomic reads did not match the reference codon. We considered each such

133 position to be a single codon variant (SCV). Analogous to single nucleotide variants (SNVs), which

134 quantify the frequency that each nucleotide allele $(A, C, G, T)$ is observed in the reads aligning to

135 a nucleotide position, SCVs quantify the frequency that each codon allele (AAA, ..., TTT) is

136 observed in the reads aligning to a codon position (see Methods for a more complete description).

137 Since SCVs are defined to be 'in-frame', they provide inherent convenience when relating

138 nucleotide variation in the genomic coordinates to amino acid variation in the corresponding

139 protein coordinates, as well as for determining whether or not nucleotide variation leads to

140 synonymous or nonsynonymous change. Within the $1 \mathrm{a} .3 . \mathrm{V}$ core genes, we found a total of

$1419,537,022 \mathrm{SCVs}$, or 128,879 per metagenome on average. These SCVs distributed throughout

142 the genome such that $78 \%$ of codons (32\% of nucleotides) exhibited minor allele frequencies

$143>10 \%$ in at least one metagenome. Despite this extraordinary level of diversity, our read

144 recruitment strategy is stringent and yields reads that on average differ from HIMB83 in only 6

145 nucleotides out of 100 (Table S2), precluding the possibility that this diversity is generated from

146 excessive nonspecific mapping. While puzzling, this level of diversity is not surprising as it agrees

147 with numerous studies that have pointed out the astonishing complexity of the SAR11 subclade 
bioRxiv preprint doi: https://doi.org/10.1101/2022.03.02.482602. this version posted March 2 2022. The copyright holder for this preprin (which was not certified by peer review) is the author/funder, who has granted bioRxiv a license to display the preprint in perpetuity. It is made available under aCC-BY-NC-ND 4.0 International license. not be further divided into sequence-discrete populations (Delmont and Kiefl et al. 2019).

150 We found this diversity to be overwhelmingly synonymous. By splitting each SCV into its 151 synonymous (s) and nonsynonymous (ns) proportions, we calculated per-site rates of s152 polymorphism and ns-polymorphism as $\mathrm{pS}^{(\text {site) }}$ and $\mathrm{pN}^{(\text {(site) }}$, not to be confused with the related 153 concepts $d S$ and $d N$. While $d S$ and $d N$ quantify rates of synonymous and nonsynonymous 154 substitution between diverged species, $\mathrm{pN}^{(\text {site) }}$ and $\mathrm{pS}^{(\text {site) }}$ can (1) resolve shorter evolutionary 155 timescales than the characteristic fixation rate, (2) be calculated from metagenomic read 156 recruitment data without complete haplotypes, and (3) define rates on a per-sample basis, thus 157 enabling inter-sample comparisons. Overall, we found that the average $\mathrm{SS}^{\text {(site) }}$ outweighed $\mathrm{pN}^{(\text {site) }}$ 158 by 19:1 (Table S3), revealing an overwhelming fraction of the 1a.3.V diversity to be synonymous 159 and illustrating how nonsynonymous mutants are purified at a much higher rate than synonymous 160 mutants in the population at large.

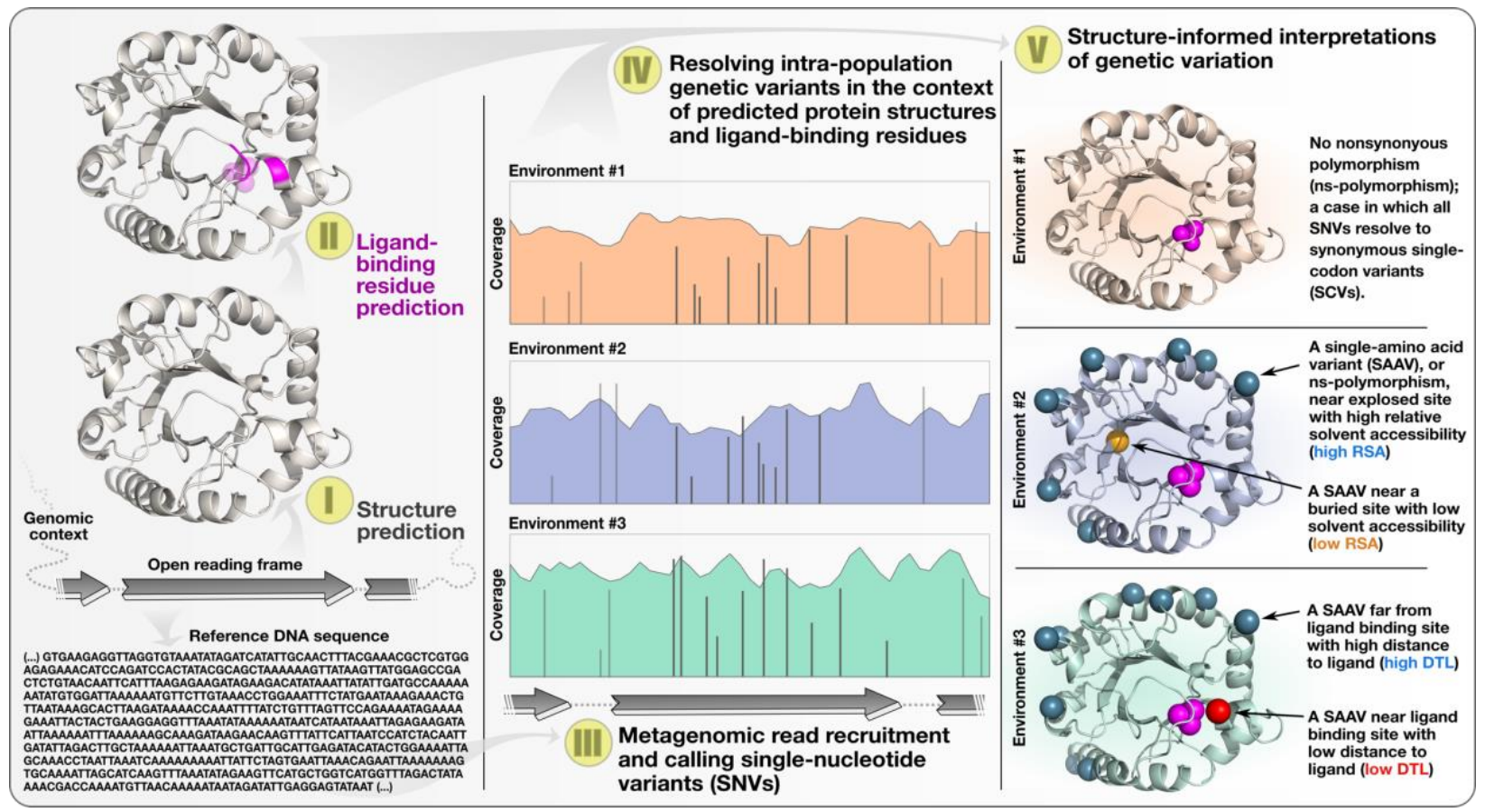

Figure 1. Anvi'o workflow for structure-informed population genetics. 


\section{Nonsynonymous polymorphism avoids buried sites}

$161 \mathrm{pN}($ site) $v a l u e s$ varied significantly from site-to-site and from sample-to-sample, but overall, more

162 variation existed between sites in a given sample than between samples of a given site (Figure

163 S3). The extent that a given site can tolerate ns-polymorphism is largely determined by the local

164 physicochemical environment of the encoded residue, which is defined by the 3D structure of the

165 protein. Thus, we broadened our focus by developing a computational framework, anvi'o structure

166 (Supplementary Information), that enabled the integration of environmental sequence variability

167 with predicted protein structures (Figure 1).

168 We used two independent methods to predict protein structures for the 799 core genes of 1a.3.V:

169 (1) a template-based homology modeling approach with MODELLER (Webb and Sali 2016), 170 which predicted 346 structures, and (2) a transformer-like deep learning approach with AlphaFold

171 (Jumper et al. 2021), which predicted 754. Our evaluation of the 339 genes for which both

172 methods predicted structures (Supplementary Information) revealed a comparable accuracy

173 between AlphaFold and MODELLER (Figure S4, Table S4). Thus, we opted to use AlphaFold

174 structures for all downstream analyses due to its higher structural coverage. Indeed, AlphaFold-

175 predicted protein structures covered over $90 \%$ of the core genes, highlighting the emerging

176 opportunities afforded by recent advances in de novo structure prediction.

177 Aligning single-codon variants to predicted structures enabled us to directly compare the 178 distributions of s-polymorphism and ns-polymorphism rates relative to biophysical characteristics 179 of the encoded proteins. We first investigated the association between polymorphism rates and 180 relative solvent accessibility $(\mathrm{RSA})$, a biophysical measure of how exposed $(\mathrm{RSA}=1)$ or buried $181(\mathrm{RSA}=0)$ a site is. Since nonsynonymous mutations at buried sites are more likely to disrupt 182 folding and stability, RSA serves as a powerful proxy to discuss the strength of structural 183 constraints acting at a site (Echave, Spielman, and Wilke 2016). By calculating RSA for each site 
184 in the predicted structures, and then weighting every site by the $\mathrm{pN}^{(\text {site) }}$ and $\mathrm{pS}^{\text {(site) }}$ across all 185 samples, we established proteome-wide distributions for $\mathrm{pN}^{(\text {site) }}$ and $\mathrm{pS}^{\text {(site) }}$ relative to RSA (Figure

186 2a). These data showed that $\mathrm{pS}^{(\text {site) }}$ closely resembled the null distribution, which illustrates the

187 lack of influence of RSA on s-polymorphism, while $\mathrm{pN}^{\text {(site) }}$ deviated significantly and instead 188 exhibited strong preference for sites with higher RSA. This finding aligns well with the expectation 189 that buried sites are likely to purify nonsynonymous change due to disruption of protein stability 190 while being relatively more tolerant to synonymous change, and validates our methodology.

\section{Nonsynonymous polymorphism avoids active sites}

191 While structural constraints ensure a given protein folds properly and remains stable, they do not 192 guarantee its function. Comprehensive analyses of diverse protein families show that residues 193 that bind or interact with ligands are depleted of mutations (Kobren and Singh 2019) due to strong 194 selective pressures that maintain active site conservancy. This constraint is not limited to the 195 immediate vicinity of ligand-binding residues, and has been observed to radiate outwards from 196 the active site with a strength inversely correlated with distance from active site (Dean et al. 2002). 197 We considered this distance as the 'distance-to-ligand' (DTL), and hypothesized that DTL may be 198 a suitable proxy for investigating functional constraints in a manner complementary to RSA, a 199 proxy for investigating structural constraints. To test this, we investigated distributions of $\mathrm{pN}^{(\text {site })}$ 200 and $\mathrm{pS}^{(\text {site) }}$ as a function of DTL for each predicted structure by first predicting sites implicated in 201 ligand binding using InteracDome (Kobren and Singh 2019), and then calculating a DTL for each 202 site, given the closest predicted ligand-binding site (Table S5). 


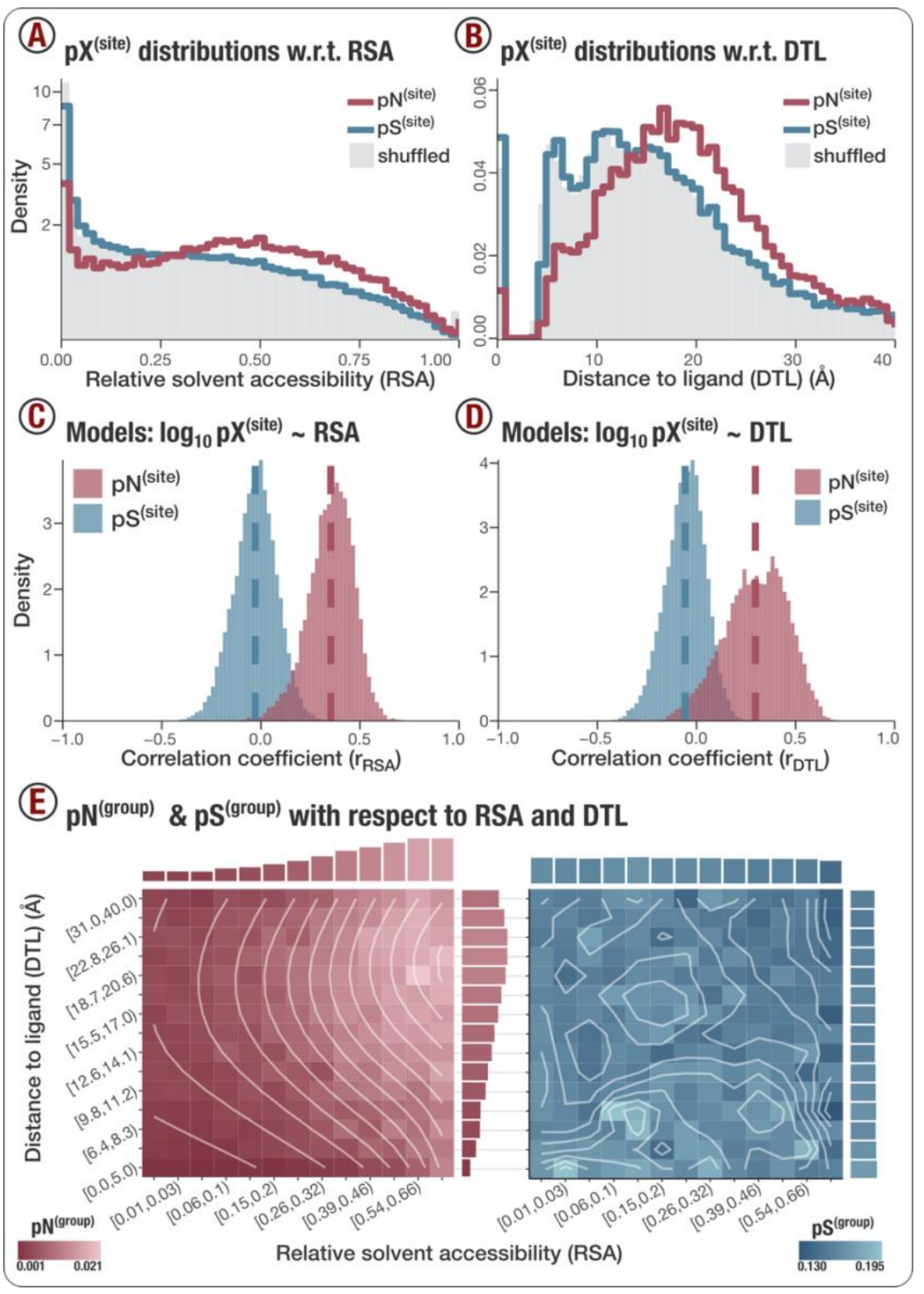

Figure 2. (A) Structural constraints shift the $\mathrm{pN}^{\text {(site) }}$ distribution towards high relative solvent accessibility (RSA). The $\mathrm{pN}$ (site) distribution (red line) and pS(site) distribution (blue line) were created by weighting the RSA values of 239,528 sites (coming from the 754 genes with predicted structures) by the $\mathrm{pN}^{\text {(site) }}$ and $\mathrm{pS}^{\text {(site) }}$ values observed in each of the 74 samples, totaling 17,725,072 pN(site) and $\mathrm{pS}^{\text {(site) }}$ values. The average distribution of 10 independent, randomly shuffled datasets of $\mathrm{pN}^{\text {(site) }}$ is depicted by the greyregions for $\mathrm{pN}$ (site), and represents the null distribution expected if no association between $\mathrm{pN}^{(\text {site })}$ and RSA existed. Since the null distribution for $\mathrm{pS}^{\text {(site) }}$ so closely resembles the null distribution for $\mathrm{pN}^{\text {(site) }}$, it has been excluded for visual clarity, but can be seen in Figure S5. (B) Functional constraint shifts the $\mathrm{pN}^{\text {(site) }}$ distribution towards high distance-to-ligand (DTL) values. The $\mathrm{pN}^{\text {(site) }}$ distribution (red line) and $\mathrm{pS}$ (site) distribution (blue line) were created by weighting the DTL values of 155,478 sites (coming from 415 genes that had predicted structures and at least one predicted ligand) by the $\mathrm{pN}^{\text {(site) }}$ and $\mathrm{pS}{ }^{(\text {site) }}$ values observed in each of the 74 samples, totaling $11,505,372 \mathrm{pN}^{\text {(site) }}$ and $\mathrm{pS}^{\text {(site) }}$ values. The $\mathrm{pN}^{\text {(site) }}$ null distribution was calculated according to the procedure described in panel $\mathrm{A}$, where again, the $\mathrm{pS}^{\text {(site) }}$ null distribution closely resembled the $\mathrm{pN}$ (site) null distribution, and can be seen in Figure S5. (C) Linear models reveal positive correlations between $\mathbf{p N} \mathbf{N}^{\text {(site) }}$ and RSA. The two distributions show Pearson correlation coefficients produced by linear models of the form $\log _{10}(\mathrm{pN}$ (site) $) \sim R S A$ (red-filled region) and $\log _{10}\left(\mathrm{pS}^{(\text {site) })} \sim \mathrm{RSA}\right.$ (blue-filled region). A model has been fit to each gene-sample pair that passed filtering criteria (see Supplementary Information), resulting in 16,285 nonsynonymous models and 24,553 synonymous models. Distribution means are visualized as dashed lines. (D) Per-group polymorphism rates explain the major selective pressure trends with respect to RSA and DTL. The left and right panels show heatmaps of $\mathrm{pN}$ (group) and $\mathrm{pS}$ (group). Each cell represents a group defined by RSA and DTL ranges shown on the $x$ - and $y$ - axes, respectively. The color of each cell represents the respective value for the group, where dark refers to low values and light refers to high values. White lines show the contour lines of smoothed data. 
203 The average per-site ns-polymorphism rate throughout the $1 \mathrm{a} .3 . \mathrm{V}$ core genome was 0.0088 ,

204 however, we observed a nearly 4 -fold reduction in this rate to just 0.0024 at predicted ligand

205 binding sites $(D T L=0)$, indicating stronger purifying selection at ligand-binding sites (Figure 2b).

206 Sites neighboring ligand-binding regions also harbored disproportionately low rates of ns-

207 polymorphism, as indicated by the significant deviation towards larger DTL values. This illustrates

208 that purifying selection that preserves proper ligand-binding functionality is not limited to residues

209 at ligand-binding sites, but extends to proximal sites as well. When we defined DTL in sequence

210 space rather than Euclidean space, this effect was no longer observable beyond sequence

211 distances of $\sim 5-10$ amino acids (Figure S6). Comparatively, $\mathrm{pS}^{\left({ }^{(s i t e)}\right.}$ deviated minimally from the

212 null distribution. Overall, integrating predicted protein structures and ligand-binding sites into the

213 analysis of the genetic diversity of an environmental population has enabled us to demonstrate

214 that (1) structural constraints bias $\mathrm{pN}^{\text {(site) }}$ distributions towards solvent exposed sites (i.e. high

$215 \mathrm{RSA}$ ) (Figure 2a), and (2) functional constraints bias $\mathrm{pN}^{(\text {site) }}$ distributions towards sites that are

216 distant from ligand-binding sites (i.e. high DTL) (Figure 2b).

\section{Proteomic trends in purifying selection are explained by RSA and DTL}

217 Given the clear shift in ns-polymorphism rates towards high RSA and DTL sites across genes, we 218 next investigated the extent that RSA and DTL can predict per-site polymorphism rates. By fitting 219 a series of linear models to log-transformed polymorphism data (Table S6), we conclude that RSA 220 and DTL can explain $11.83 \%$ and $6.89 \%$ of $\mathrm{pN}^{(\text {site) }}$ variation, respectively. Based on these models 221 we estimate that for any given gene in any given sample, (1) a 1\% increase in RSA corresponds 222 to a $0.98 \%$ increase in $\mathrm{pN}^{(\text {site) }}$, and (2) a $1 \%$ increase in DTL (normalized by the maximum DTL in 223 the gene) corresponds to a $0.90 \%$ increase in $\mathrm{pN}^{(\text {site) }}$. In a combined model, RSA and DTL jointly 224 explained $14.12 \%$ of $\mathrm{pN}^{(\text {site) }}$ variation, and after adjusting for gene-to-gene and sample-to-sample 
variance, $17.07 \%$ of the remaining variation could be explained by RSA and DTL. In comparison, only $0.35 \%$ of $\mathrm{pS}^{(\text {site) }}$ variation was explained by RSA and DTL. Using a complementary approach, we constructed models for each gene-sample pair (Supplementary Information), the correlations of which we used to visualize the extent that $\mathrm{pN}^{(\text {site) }}$ can be modeled by RSA and DTL relative to $\mathrm{pS}^{(\text {site) }}$ (Figures 2c, 2d). Analyzing gene-sample pairs revealed that the extent of ns-polymorphism rate that can be explained by RSA and DTL is not uniform across all genes (Table S7) and can reach up to 52.6\% and 51.4\%, respectively (Figures S7, S8). Finally, we averaged polymorphism rates within groups of sites that shared similar RSA and DTL values, which demonstrated the tight association between the rate of within population ns-polymorphism rate and protein structure

234 (Table S8, Figure 2e). Linear regressions of these data show that $83.6 \%$ of per-group nspolymorphism rates and $20.7 \%$ of per-group s-polymorphism rates are explained by RSA and DTL (Supplementary Information).

237 The true predictive power of RSA and DTL for polymorphism rates is most likely higher than we 238 report, since our approaches suffer from methodological shortcomings. For instance, we calculate 239 RSA from the steric configurations of residues in predicted structures. Thus, errors in structure 240 prediction propagate to errors in RSA. Errors in structure also propagate to errors in DTL, since

241 DTL is calculated using Euclidean distances between residues, which is exacerbated by the 242 uncertainty associated with ligand-binding site predictions. Furthermore, RSA and DTL 243 calculations assume that the protein is monomeric, even though oligomeric proteins are common, 244 and they represent the majority of proteins in some organisms (Goodsell and and Olson 2003). 245 In these cases, exposed sites in the monomeric structure could be buried once assembled into 246 the quaternary structure, and this is similarly true for estimates of DTL. Even if we assume 247 structural predictions are $100 \%$ accurate, it is notable that binding site predictions exclude (1) 248 ligands that are proteins, (2) ligand-protein complexes that have not co-crystallized with each 249 other, (3) ligands of proteins with no shared homology in the InteracDome database, and (4) 
250 unknown ligand-protein complexes. Each of these shortcomings leads to missed binding sites,

251 which leads to erroneously high DTL values in the proximity of unidentified binding sites (Figure

252 S9). Furthermore, our predictions assume that if a homologous protein in the InteracDome

253 database binds to a ligand with a particular residue, then so too does the corresponding residue

254 in the HIMB83 protein. This leads to uncertain predictions, since homology does not necessitate

255 binding site conservancy. Yet, despite these methodological shortcomings, our analyses show

256 that RSA and DTL are significant predictors of per-site and per-group variation.

257 Clear partitioning of environmental genetic variation by RSA and DTL (Figure 2) highlights the

258 utility of these metrics for studies of evolution following the increasing availability of protein

259 structures. Analyses of total genetic variation lacking the ability to delineate distinct processes of

260 evolution limit opportunities to identify determinants of fitness in rich and complex data afforded

261 by environmental metagenomes. Indeed, the application of RSA and DTL to SAR11 demonstrate

262 that not all variants are created equal; a notion considered common knowledge by all life

263 scientists, and yet such a treatment is lacking in studies of genomic heterogeneity that rely upon

264 metagenomic read recruitment. RSA and DTL provide quantitative means to bring a level of

265 scrutiny to distinguish variants based on their distributions in proteins. For instance, a collection

266 of high-RSA and high-DTL sites will be more likely to be enriched in neutral variants. In contrast,

267 residues under strong purifying selection will more likely be enriched in low-RSA and/or low-DTL

268 sites of proteins. The ability to tease apart distinct evolutionary processes with absolute accuracy

269 will indeed remain difficult due to a multitude of factors. But by providing structure-informed means

270 to partition the total intra-population variation into distinct pools, RSA and DTL offer a quantitative

271 framework that enables new opportunities to study distinct evolutionary processes. 


\section{Measuring purifying selection between genes and environments with $\mathrm{pN} / \mathrm{pS}$ (gene)}

272 So far, our structure-informed investigation has focused on trends of sequence variation within 273 the gene pool of an environmental population. Next, we shifted our attention to individual proteins. $274 \mathrm{pN} / \mathrm{pS}^{(\text {gene) }}$ is a metric that quantifies the overall direction and magnitude of selection acting on a 275 single gene (Schloissnig et al. 2013; Shenhav and Zeevi 2020), where pN/pS ${ }^{(\text {gene) }}<1$ indicates 276 the presence of purifying selection, the intensity of which increases as the ratio decreases. Since

$277 \mathrm{pN} / \mathrm{pS}^{(\text {gene) }}$ is defined for a given gene in a given sample, $\mathrm{pN} / \mathrm{pS}{ }^{(\text {gene) }}$ values for a single gene can 278 be compiled from multiple samples, enabling the tracking of selective pressures across 279 environments (Shenhav and Zeevi 2020). Taking advantage of the large number of metagenomes 280 in which 1a.3.V was present, we calculated $\mathrm{pN} / \mathrm{pS}^{(\mathrm{gene})}$ for all 799 protein-coding core genes 281 across 74 samples (see Methods), resulting in 59,126 gene/sample pairs (Table S9). We 282 validated our calculations by comparing sample-averaged $\mathrm{pN} / \mathrm{pS}^{\text {(gene) }}$ to $\mathrm{dN} / \mathrm{dS}{ }^{\text {(gene) }}$ calculated 283 from homologous gene pairs between HIMB83 and HIMB122, another SAR11 isolate genome 284 that is closely related to HIMB83 (gANI: $82.6 \%$ ), which we found to yield commensurable results 285 (Figure S10, Table S12, Supplementary Information).

286 We found significantly more $\mathrm{pN} / \mathrm{pS}^{\text {(gene) }}$ variation between genes of a given sample ('gene-to287 gene' variation) than between samples of a given gene ('sample-to-sample' variation) (ANOVA, 288 Figure S11). All but one gene (gene \#2031, unknown function) maintained pN/pS ${ }^{(g e n e)}<1$ in 289 every sample, whereby $95 \%$ of values were less than 0.15 (Figure S12, Table S9), indicating an 290 intense purifying selection for the vast majority of 1a.3.V genes across environments. This was 291 foreshadowed by our earlier analysis in which $\mathrm{pS}^{\text {(site) }}$ outweighed $\mathrm{pN}^{(\text {site) }}$ by $19: 1$ within the 292 aggregated data across genes and samples. However, the magnitude of purifying selection was 293 not uniform across all genes. In fact, gene-to-gene variance, as opposed to sample-to-sample 294 variance, explained $93 \%$ of $\mathrm{pN} / \mathrm{pS}^{\text {(gene) }}$ variation (ANOVA, Figure S11). By analyzing the 
companion metatranscriptomic data (Salazar et al. 2019) that were available for 50 of the 74

metagenomes, we were able to explain $29 \%$ of gene-to-gene variance with gene transcript abundance (Table S13, Supplementary Information), a known predictor of evolutionary rate (Pál,

298 Papp, and Hurst 2001). Overall, these data demonstrate the utility of $\mathrm{pN} / \mathrm{pS}^{(\text {gene) }}$ as a metric to 299 understand the overall extent of selection acting on genes.

300 The amount of $\mathrm{pN} / \mathrm{pS}^{\text {(gene) }}$ variation attributable to sample-to-sample variance was only $0.7 \%$

301 (Figure S11). While it represents a small proportion of the total variance, the sample-to-sample 302 variance in $\mathrm{pN} / \mathrm{pS}^{\left({ }^{(g e n e)}\right.}$ encapsulates the extent that polymorphism varies in response to the range 303 of environmental parameters observed across samples. These data therefore provide the 304 opportunity to relate how differences in genetic diversity of individual genes manifests from 305 differences in environmental parameters (Table S10), which we focused on next.

\section{Nitrogen availability governs rates of non-ideal polymorphism at critical sites of glutamine synthetase}

306 To gain a more highly resolved picture of how selection shapes protein evolution, we searched

307 for a biologically relevant gene within 1a.3.V that exhibited evolutionary patterns that could be

308 understood by leveraging structural information. Glutamine synthetase (GS) is a critical enzyme

309 for the recycling of cellular nitrogen (Bernard and Habash 2009), a limiting nutrient for microbial

310 productivity in surface oceans (Bristow et al. 2017). GS yields glutamine and ADP from glutamate,

311 ammonia, and ATP, an essential step in the biosynthesis of nitrogenous compounds.

312 Given the central role that GS plays in nitrogen metabolism, we expected GS to be under high

313 selection. Indeed, the sample-averaged $\mathrm{pN} / \mathrm{pS}^{(\mathrm{GS})}$ was 0.02 , ranking $\mathrm{GS}$ amongst the top $11 \%$

314 most purified genes (Figure 3b, Table S9). Although highly purified, we observed significant 315 sample-to-sample variation in $\mathrm{pN} / \mathrm{pS}^{(\mathrm{GS})}(\min =0.010, \max =0.036)$ suggesting that the strength 316 of purifying selection on GS varies from sample to sample (Figure $3 b$ inset), perhaps due to unique 
317 environmental conditions (e.g., nutrient compositions) that differentially impact the need for

318 glutamine synthesis. Since previous work has shown that SAR11 upregulates its transcriptional 319 and translational production of GS in response to nitrogen limitation (Smith Daniel P. et al., n.d.), 320 we hypothesized that purifying selection should be highest in nitrogen-limited environments, and 321 lowest in nitrogen-replete environments. We utilized measured concentrations of nitrate as an 322 indication of the level of nitrogen limitation in each sample, and found a positive correlation 323 between measured nitrate concentrations and $\mathrm{pN} / \mathrm{pS}^{(\mathrm{GS})}$ values across samples (Pearson 324 correlation $p$-value $\left.=0.009, R^{2}=0.11\right)($ Figure $3 c)$, which ranked amongst the top $12 \%$ of positive 325 correlations between $\mathrm{pN} / \mathrm{pS}^{\text {(gene) }}$ and nitrate concentration (Figure $3 \mathrm{c}$ inset, Table S10). In 326 summary, we find that although GS is under high selection, subtle differences in selection strength are observed between samples and are most likely driven by nitrogen availability.

328 Next, we focused on the GS protein structure to further investigate the associations between GS 329 polymorphism and processes of selection. Since the native quaternary structure of GS is a 330 dodecameric complex (12 monomers), our monomeric estimates of RSA and DTL are 331 unrepresentative of the active state of GS. We addressed this by aligning 12 copies of the 332 predicted structure to a solved dodecameric complex of GS in Salmonella typhimurium (PDB ID 333 1FPY), which HIMB83 GS shares $61 \%$ amino acid similarity with (Figure 3a). From this stitched 334 quaternary structure we recalculated RSA and DTL, and as expected, this yielded lower average 335 RSA and DTL estimates due to the presence of adjacent monomers ( 0.17 versus 0.24 for RSA and $17.8 \AA$ versus $21.2 \AA$ for $D T L)$. With these quaternary estimates of RSA and DTL, we found 337 that ns-polymorphism was 30x less common than s-polymorphism, and it strongly avoided sites 338 with low RSA and the three glutamate active sites to which any given monomer was proximal 339 (Figure 3d). In comparison, s-polymorphism distributed relatively homogeneously throughout the 340 protein, whereby $17 \%$ of s-polymorphism occurred within $10 \AA$ of active sites (compared to $3 \%$ for 341 ns-polymorphism) and 19\% occurred in sites with 0 RSA (compared to 9\% for ns-polymorphism). 
342 Averaged across samples, the mean RSA was 0.15 for s-polymorphism and 0.33 for ns-

343 polymorphism (Figure $3 e$ left panel). Similarly, the mean DTL was $17.2 \AA$ for s-polymorphism and

$34422.9 \AA$ for ns-polymorphism (Figure $3 f$ left panel). These observations highlight in a single gene

345 what we previously observed across the 1a.3.V core: selection purifies the majority of ns-

346 polymorphism and does so with increased strength at structurally/functionally critical sites.

347 We next investigated whether variance in selection strength (Figure 3b inset) affects the spatial

348 distribution patterns of polymorphism. For each sample, we calculated how polymorphism rates

349 in GS distributed with respect to RSA and DTL and associated these distributions with $\mathrm{pN} / \mathrm{pS}^{(\mathrm{GS})}$.

350 While the mean RSA of s-polymorphism remained relatively invariant (standard deviation 0.005 )

351 (Figure 3e right panel), the mean RSA of ns-polymorphism varied dramatically from 0.27 to 0.37

352 and was profoundly influenced by sample $\mathrm{pN} / \mathrm{pS}^{(\mathrm{GS})}$; samples exhibiting low selection of GS

353 harbored lower mean RSA and samples exhibiting high selection of GS harbored higher mean

354 RSA (Figure 3e right panel). In fact, $82.9 \%$ of mean RSA ns-polymorphism variance could be

355 explained by $\mathrm{pN} / \mathrm{pS}^{(\mathrm{GS})}$ alone (Pearson correlation, $\mathrm{p}$-value $<1 \times 10^{-16}, \mathrm{R}^{2}=0.829$ ). ns-

356 polymorphism distributions with respect to DTL were equally governed by selection strength,

357 where $80.4 \%$ of variance could be explained by $\mathrm{pN} / \mathrm{pS}{ }^{\text {(GS) }}$ (Pearson correlation, $\mathrm{p}$-value $<1 \times 10^{-}$

$358{ }^{16}, R^{2}=0.804$, Figure $\left.3 f\right)$. 

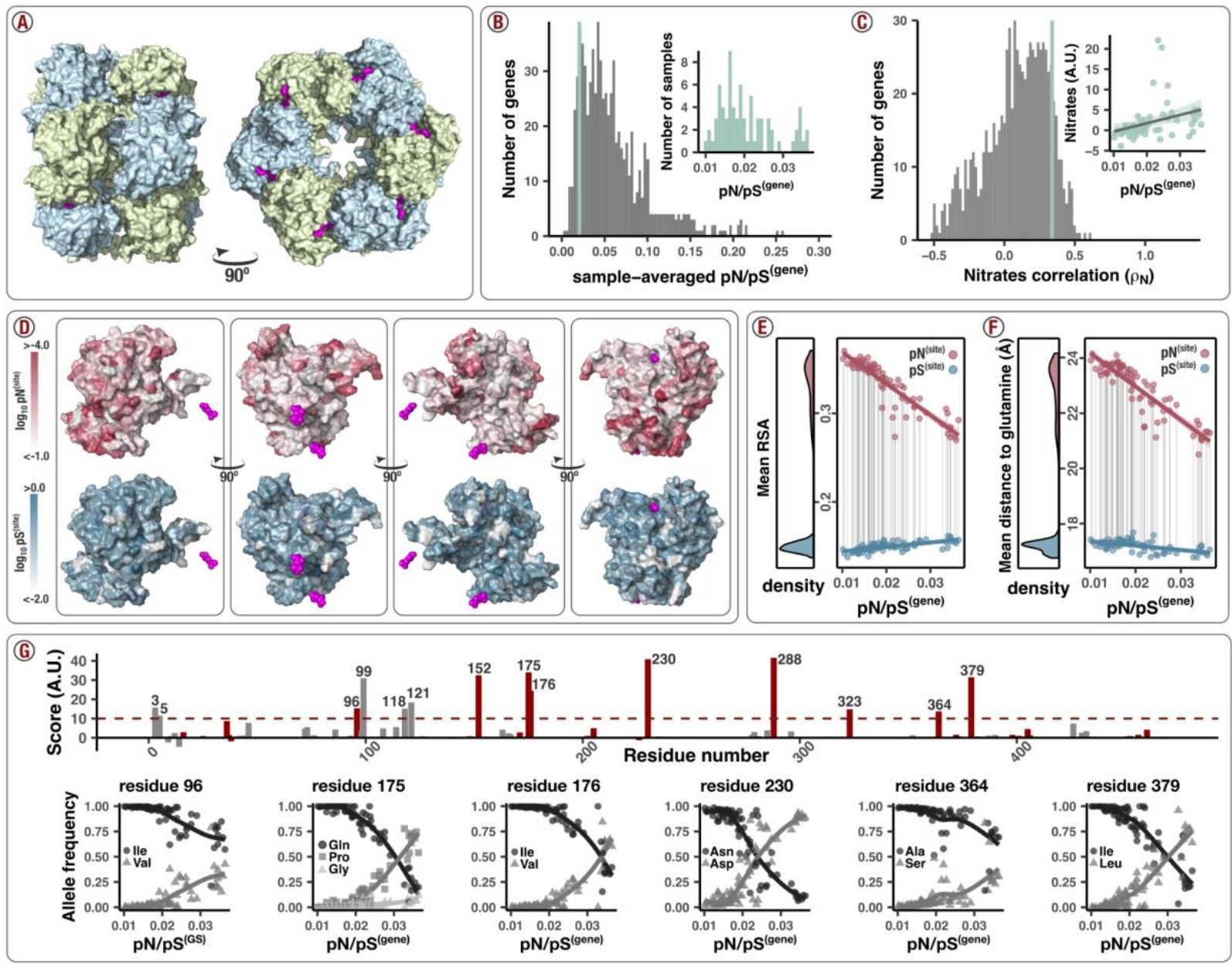

Figure 3. Polymorphism distribution patterns in glutamine synthetase (GS). (A) GS forms a dodecameric complex. The structure (PDB ID 1FPY) comes from Salmonella typhimurium (61\% sequence similarity to HIMB83) and is shown from two different views. Pink molecules are ADP and phosphinothricin (steric inhibitor of glutamate), and are situated within the active site of GS. (B) GS is one of the most highly conserved genes in 1a.3.V. The main plot shows the distribution of sample-averaged $\mathrm{pN} / \mathrm{pS}^{\text {(gene) }}$ for all 799 genes in the 1a.3.V core (truncated at 0.30). The vertical green line depicts the sample-averaged $\mathrm{pN} / \mathrm{pS}^{(\text {gene) }}$ for GS $(0.020)$. The inset plot shows the distribution of $\mathrm{pN} / \mathrm{pS}$ (gene) value for GS as seen across the 74 samples, which vary from 0.010 to 0.036 . (C) Selection strength on GS correlates with environmental concentration of nitrates. The main plot shows a histogram of Pearson correlation coefficients (one per gene) between $\mathrm{pN} / \mathrm{pS}^{\text {(gene) }}$ and measured concentration of nitrates in each sample. The vertical green line depicts the correlation coefficient for GS (0.34). The inset shows a scatter plot of $\mathrm{pN} / \mathrm{pS}$ (gene) vs nitrate concentrations from which the GS correlation coefficient was calculated. (D) ns-polymorphism polymorphism rates are reduced in the vicinity of the active sites. Each image is a view of the predicted structure of monomeric GS. Phosphinothricin substrates were situated by aligning the predicted GS structure to the complex in panel A. Red surfaces are colored according to the sample-averaged $\log _{10} \mathrm{p} N($ site) value of each residue, and blue surfaces are colored according to the sample-averaged $\log _{10} \mathrm{pS}^{(\text {site) }}$ value of each residue. In each case, darker colors refer to higher rates. Left-to-right, each view is a $90^{\circ}$ clockwise rotation of the previous view about the vertical axis. Each image was rendered programmatically using a PyMOL script that was generated from the anvi'o structure interactive interface. (E) As 
selection decreases, ns-polymorphism creeps into low-RSA sites. The left panel shows the distribution of samples' average RSA of nonsynonymous (red) and synonymous (blue) polymorphisms. The right panel shows how these average RSA values ( $y$-axis) correlate with the samples' $\mathrm{pN} / \mathrm{pS}^{\text {(gene) }}$ values ( $\mathrm{x}$-axis). Each data point is calculated by weighting the RSA of each residue by the $\mathrm{pN}^{\text {(site) }}$ (red) or $\mathrm{pS}^{\text {(site) }}$ (blue) values observed in that sample. The red and blue lines show the nonsynonymous and synonymous linear fits, respectively, and the corresponding shaded regions show the $95 \%$ confidence intervals for the fit. (F) As selection decreases, ns-polymorphism creeps closer to the binding site. The scheme is identical to panel E, where RSA is replaced with the distance-to-glutamate substrate (DTL). (G) Some sites exhibit amino acid minor allele frequencies that co-vary with $\mathbf{p N} / \mathbf{p S}^{(\mathrm{GS})}$. The top panel shows the extent that sites co-vary with $\mathrm{pN} / \mathrm{pS}^{(\mathrm{GS})}$. The $\mathrm{x}$-axis shows the residue number and the $\mathrm{y}$-axis the slope estimate of a linear regression between the sum of minor allele frequencies and $\mathrm{pN} / \mathrm{pS}$ (GS). Sites with $\mathrm{DTL}$ values less than the average are indicated in red and are gray otherwise. All sites above an arbitrary cutoff (dashed horizontal line) are annotated with their residue number. Scatter plots below show the allele frequency trajectories for a select number of these sites.

When selection is low, we observe high nitrate concentrations (Figure $3 c$ inset) and nspolymorphism distributions towards lower RSA/DTL (Figures 3e, 3f). When selection is high, we observe low environmental nitrate concentrations (Figure $3 \mathrm{c}$ inset) and ns-polymorphism distributions towards higher RSA/DTL (Figures 3e, 3f). Given that proper functionality of GS is most critical in nitrogen-limited environments and that mutations with low RSA/DTL are more likely

364 to be deleterious, the most likely explanation for the body of evidence presented is that GS 365 accumulates non-ideal polymorphism in samples exhibiting low selection of GS that cannot be 366 effectively purified at the given selection strength. As selection increases, so too does the 367 purifying efficiency, which we indirectly measure as increases in mean RSA and DTL of ns368 polymorphism. Our approach illustrates this 'use it or lose it' evolutionary principle over a spectrum of selection strengths which have been sampled from natural in situ environmental conditions.

371 Under this hypothesis, there should exist low DTL amino acid alleles that create a negative, yet 372 tolerable impact on fitness when selection is low, yet incur an increasingly detrimental fitness cost 373 as selection increases. One would expect such alleles to be at low frequency in low $\mathrm{pN} / \mathrm{pS}^{(\mathrm{GS})}$ 374 samples, and to reach increasingly higher frequencies in higher $\mathrm{pN} / \mathrm{pS}^{(\mathrm{GS})}$ samples. We identified 375 putative sites fitting this description by scoring sites based on the extent that their amino acid 
376 minor allele frequencies co-varied with $\mathrm{pN} / \mathrm{pS}^{(\mathrm{GS})}$, including only sites with DTL less than the mean

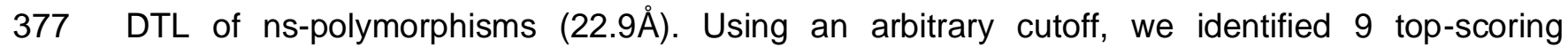
378 polymorphisms that co-varied with $\mathrm{pN} / \mathrm{pS}^{(\mathrm{GS})}$ (Figure 3g): 196V, L152I, Q175P/G, I176V, N230D, 379 S288A/D, I323V, A364S, I379L. Though each of these sites exhibited DTL lower than the average 380 ns-polymorphism, the closest site (residue number 323 ) was still $9 \AA$ away from the glutamate 381 substrate. This suggests there are no 'smoking gun' polymorphisms occurring in the binding site 382 that abrasively disrupt functionality. After all, in absolute terms GS is highly purified regardless of 383 sample - the largest $\mathrm{pN} / \mathrm{pS}^{(\mathrm{GS})}$ is 0.036 , which is just over half the genome-wide average $384 \mathrm{pN} / \mathrm{pS}^{\text {(gene) }}$ of 0.063 . Our data therefore represents a subtle, yet resolvable signal of minute decreases in selection strength manifesting as minute shifts in the distribution of ns-polymorphism towards the active site.

387 While identifying signatures of positive selection is typically the primary pursuit in evolutionary 388 analysis, our data instead illustrates a highly resolved interplay between purifying selection 389 strength and polymorphism distribution. The geography and unique environmental parameters 390 associated with each sample yielded a spectrum of selection strengths which enabled us to 391 quantify how polymorphism distributions of a gene under high selection shift in response to small 392 perturbations in selection strength. In the case of GS, we were able to attribute these shifts to the 393 availability of nitrogen, thereby linking together environment, selection, and polymorphism.

394 Throughout the 1a.3.V core genes, we observed that samples exhibiting low overall selection of 395 1a.3.V were strongly associated with increased accumulation of ns-polymorphism at low 396 RSA/DTL sites (Figures 4a, 4b, Supplementary Information), suggesting this signal is not specific 397 to GS, but rather a general feature of the 1a.3.V core genes. Though highly significant (one sided 398 Pearson p-values $9 \times 10^{-12}$ for RSA and $2 \times 10^{-4}$ for DTL), the magnitude that ns-polymorphism 399 distributions shift with respect to DTL and RSA were subtle: across samples, the mean DTL of 400 ns-polymorphism varied by less than $1 \AA$, and the mean RSA varied between 0.230 and 0.236 . 
bioRxiv preprint doi: https://doi.org/10.1101/2022.03.02.482602; this version posted March 2, 2022. The copyright holder for this preprint (which was not certified by peer review) is the author/funder, who has granted bioRxiv a license to display the preprint in perpetuity. It is made available under aCC-BY-NC-ND 4.0 International license.
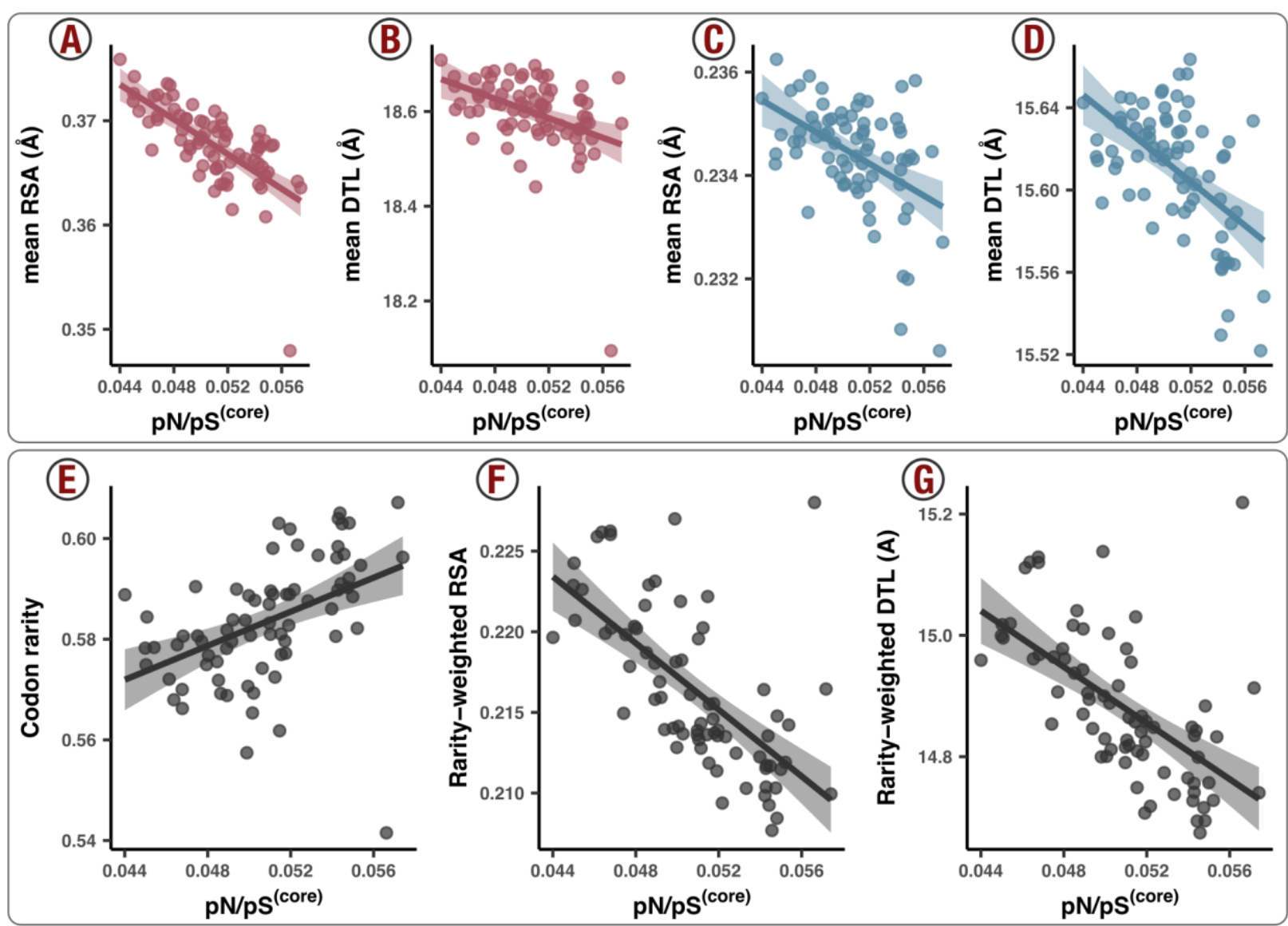

Figure 4. Polymorphism distribution patterns with respect to genome-wide selection strength. Each data point is a sample (metagenome). Lines represent lines of best fit and corresponding translucent areas represent $95 \%$ confidence intervals. The $\mathrm{x}$-axis is $\mathrm{pN} / \mathrm{pS}^{(\text {(core) }}$, which is calculated across the whole core genome and is an inverse proxy of genome-wide purifying selection strength (see Methods). (A) The ns-polymorphism distribution mean with respect to RSA is negatively associated with $\mathrm{pN} / \mathrm{pS}^{\text {(core) }}$ (one-sided Pearson $\mathrm{p}$-value $=9 \times 10^{-12}$ ). (B) The nspolymorphism distribution mean with respect to DTL is negatively associated with $\mathrm{pN} / \mathrm{pS}{ }^{\text {(core) }}$ (one-sided Pearson $\mathrm{p}$ value $=2 \times 10^{-4}$ ). (C) The s-polymorphism distribution mean with respect to RSA is negatively associated with $\mathrm{pN} / \mathrm{pS}^{\text {(core) }}$ (one-sided Pearson $p$-value $=1 \times 10^{-5}$ ). (D) The s-polymorphism distribution mean with respect to RSA is negatively associated with $\mathrm{pN} / \mathrm{pS}^{\text {(core) }}$ (one-sided Pearson $\mathrm{p}$-value $=3 \times 10^{-7}$ ). $(\mathrm{E})$ Rare synonymous codons are more abundant in samples with high $\mathrm{pN} / \mathrm{pS}^{(\text {core) }}$ (one-sided Pearson $\mathrm{p}$-value $=4 \times 10^{-5}$ ). $(\mathbf{F})$ Rare synonymous codons avoid low RSA sites when $\mathrm{pN} / \mathrm{pS}^{\text {(core) }}$ is low (one-sided Pearson $\mathrm{p}$-value $=1 \times 10^{-10}$ ). (G) Rare synonymous codons avoid low DTL sites when $\mathrm{pN} / \mathrm{pS}^{\text {(core) }}$ is low (one-sided Pearson $\mathrm{p}$-value $=7 \times 10^{-9}$ ). 


\section{Synonymous but not silent: selection against rare codons at critical sites}

403 Thus far we have observed that purifying efficiency observably decreases in response to lowered

404 selection strength, as evidenced by ns-polymorphism occurring nearer to binding sites and in

405 more buried sites. Given the influence of synonymous substitutions in translational processes

406 (Plotkin and Kudla 2011), as a final analysis we focused on within-population trends of s-

407 polymorphism.

408 Compared to ns-polymorphism, s-polymorphism distributes more uniformly throughout protein

409 structures (Figures 2a, 2b). Yet our data also revealed an association between selection strength

410 and the distribution of s-polymorphism. In samples under higher selection, s-polymorphism

411 systematically tended to occur (1) in more solvent-exposed sites (Figure 4c, one-sided Pearson

$412 p$-value $=1 \times 10^{-5}$ ) and (2) farther from binding sites (Figure 4d, one-sided Pearson $p$-value $=3 \times 10^{-}$

$413^{7}$ ). These trends indeed mimic the nonsynonymous trends in glutamine synthetase (Figures 3d,

$4143 e, 3 f)$ as well as the core genes in general (Figures 4a, 4b), and cannot be reasonably explained

415 by neutral processes. The surprising association suggests a relationship between selection and

416 synonymous change that is at least partly determined by structural features of proteins.

417 With a GC-content lower than 30\%, SAR11 genomes maintain a non-uniform yet conserved 418 codon composition (Figure S13). Previous work has shown that rare codons can significantly 419 reduce translation rates (Sørensen, Kurland, and Pedersen 1989), cause delays in the production 420 of the polypeptide chain at the ribosome (Komar 2009), which can lead to protein misfolding 421 (Drummond and Wilke 2008; Agashe et al. 2013), and impair fitness (Walsh et al. 2020). Thus, 422 we hypothesized that rare codons in 1a.3.V may incur fitness costs relative to their more common, 423 synonymous counterparts. To test this hypothesis, we investigated the relationship between 424 selection strength and the occurrence of rare codons, which required us to define a 'codon rarity' 
425 metric based on the frequency that codons are found in the HIMB83 genome relative to their 426 synonymous counterparts (Table S11). We then attributed an overall rarity score to each sample 427 by weighting the rarity of all synonymous codon alleles by the frequencies with which they were 428 observed (see Methods). Our analysis of these data revealed a positive correlation between 429 codon rarity in a sample and its $\mathrm{pN} / \mathrm{pS}^{(\text {core) }}$ (Figure $4 \mathrm{~d}$, one-sided Pearson $\mathrm{p}$-value $=1 \times 10^{-5}$ ),

430 illustrating that rare codons are more likely to be found in samples where genome-wide selection 431 is low. We found this to be the case for s-polymorphism within all 18 amino acids that possess 432 two or more codons (Figure S14), illustrating that this evolutionary process acts ubiquitously 433 throughout the genetic code of 1a.3.V. Rare codons did not distribute throughout protein 434 structures uniformly, either. In samples with low genome-wide selection, where rarity was highest, 435 rare codons occurred farther away from binding sites (one-sided Pearson $p$-value $=1 \times 10^{-10}$ ) and occurred more frequently in more solvent-exposed sites (one-sided Pearson p-value $=7 \times 10^{-9}$ ), as compared to low selection samples (Figures $4 \mathrm{e}, 4 \mathrm{f}$ ).

438 Overall, these data show that when genome-wide selection strength is low, rare codons both (1) 439 incorporate into the genome with increased propensity, and (2) manifest in sites that are 440 statistically more likely to be structurally/functionally important. As previous research suggests, 441 the most likely explanation for these observations is that rare codons are less fit due to decreased 442 translational accuracy compared to their more common, synonymous counterparts. Yet the 443 environmental and structural dimensions of our data reveal the dynamic nature of the evolutionary 444 processes that maintain synonymous polymorphism as a function of changing conditions in 445 naturally occurring habitats and elucidates the intensity of such processes as a function of their 446 physical locations in the structure. Indeed, 1a.3.V maintains the lowest proportion of rare codons 447 in samples where genome-wide selection is highest, and rare codons in these samples are 448 statistically more likely to be incorporated in noncritical sites of proteins, most likely due to the 449 increased efficiency with which purifying selection operates in an environment- and site- 
bioRxiv preprint doi: https://doi.org/10.1101/2022.03.02.482602; this version posted March 2, 2022. The copyright holder for this preprint (which was not certified by peer review) is the author/funder, who has granted bioRxiv a license to display the preprint in perpetuity. It is made available under aCC-BY-NC-ND 4.0 International license.

450 dependent manner. These rare codon data provide a lens into the potential fitness costs

451 associated with suboptimal translational accuracy in complex populations, and by including

452 structural data, we demonstrate where optimal translational accuracy matters most. 


\section{Conclusions}

453 With recent breakthroughs in predicting protein structures and ligand binding sites, microbial

454 ecology need not be limited to just sequences. By offering an interactive, scalable, and open-

455 source software solution that integrates environmental genetic variants with structural

456 bioinformatics, our study takes advantage of recent advances to connect environmental 'omics

457 and structural biology. Indeed, by leveraging structure and ligand-binding predictions we were

458 able to describe striking patterns of nucleotide polymorphism in an environmental microbial

459 population that we could ascribe to evolutionary constraints that preserve protein structure (folding

$460 \&$ stability) and protein function (ligand-binding activity). By tracking a SAR11 population across

461 metagenomes we were able to demonstrate the presence of dynamic processes that purge both

462 synonymous and nonsynonymous polymorphism from the vicinity of ligand binding sites of

463 proteins as a function of selection strength. Overall, our study proposes a structure-informed

464 computational framework for microbial population genetics and offers a glimpse into the emerging

465 interdisciplinary opportunities made available at the intersection of ecology, evolution, and

466 structural biology. 


\section{Methods}

467 Overview. The URL https://merenlab.org/data/anvio-structure/ provides a complete reproducible

468 workflow for all analysis steps detailed below, including (1) downloading the publicly available

469 metagenomes and genomes, (2) recruiting reads from metagenomes, (3) calculating single

470 amino-acid and single codon variants, (4) predicting protein structures and ligand binding sites,

471 and (5) visualizing metagenomic sequence variants and binding sites onto protein structures.

472 Metagenomic and metatranscriptomic read recruitment and processing. To study the

473 population structure of the environmental SAR11 population 1a.3.V defined previously (Delmont

474 and Kiefl et al. 2019), we used anvi'o v7.1 (Eren et al. 2021), and its metagenomics workflow

475 (Shaiber et al. 2020) which uses snakemake v5.10 (Köster and Rahmann 2012) to automate gene

476 calling, gene function annotation, metagenomic and metatranscriptomic read recruitment steps.

477 The compendium of anvi'o programs the metagenomics workflow called upon employed Prodigal

478 v2.6.3 (Hyatt et al. 2010) for gene calling, NCBl's Clusters of Orthologous Groups (COGs)

479 database (Tatusov et al. 2003) and Pfams (El-Gebali et al. 2019) for gene function annotation,

480 HMMER v3.3 (Eddy 2011) for profile HMM searches, DIAMOND v2.0.6 (Buchfink, Xie, and Huson

481 2015) for sequence searches, Bowtie2 v2.4 (Langmead and Salzberg 2012) for read recruitment,

482 and samtools v1.9 (Li et al. 2009) to generate BAM files. The metagenomic workflow resulted in 483 a 'contigs database' and a 'merged profile database' (two anvi'o artifacts detailed at

484 https://anvio.org/help/), which gives access to gene and genome coverages (with metagenomic 485 or metatranscriptomic short reads), as well as the sequence variability data to study population 486 genetics as detailed below. We adopted a competitive read recruitment strategy by using all 487 SAR11 genomes, rather than only HIMB83, as reference to recruit reads from Tara Oceans 488 Project metagenomes and metatranscriptomes to maximize the exclusion of reads that matched 489 better to other known SAR11 genomes, thereby narrowing our scope of probed diversity and 
minimizing the impacts of non-specific read recruitment. In all subsequent analyses we focused on the core genes of the 1a.3.V subclade by only considering (a) reads that mapped to HIMB83 (b) the 74 metagenomes in which HIMB83 was found above 50X, and (c) the 799 HIMB83 genes

493 that were previously found to maintain consistent coverage patterns (Delmont and Kiefl et al. 494 2019).

Quantifying SCVs and SAAVs in metagenomes. To characterize the variants in metagenomic read recruitment results we used and extended the microbial population genetics framework 497 implemented in anvi'o. The program `anvi-profile` with the flag '--profile-SCVs` characterizes 498 single codon variants (SCVs), from which single amino acid variants (SAAVs) can also be 499 calculated. Anvi'o determines allele frequency vectors for SCVs by tallying the frequencies of 500 codons observed in the 3-nt segments of reads that fully map to a given codon position. The 501 frequencies of amino acids encoded by each 3-nt segment yield SAAVs observed in a given 502 position, which represent allele frequency vectors of positions after collapsing synonymous 503 redundancy. For a given codon position, anvi'o excludes any reads that do not map to all 3 504 nucleotides, which can happen either if the read terminates within the codon position, or there 505 exists a deletion in the read relative to the reference genome. Reads that contain insertions within 506 the codon relative to the reference genome are also excluded during this step. We exported 507 variant profiles as tabular data using the program `anvi-gen-variability-profile`, where each row is 508 a SCV (or SAAV) and the columns specify (1) identifying information such as the corresponding 509 gene, codon position, and sample id, (2) the number of mapped reads corresponding to each of 510 the 64 codons (or 20 amino acids), and (3) numerous miscellaneous statistics, all of which can 511 be explored at https://merenlab.org/analyzing-genetic-varaibility/.

512 Calculations of polymorphism rates of individual codon sites, $\mathrm{pN}^{(\text {(ite) }}$ and $\mathrm{pS}^{\text {(site) }}$. We 513 calculated the polymorphism rates of individual codon sites from allele frequencies defined from 514 each SCV based on a recent study by Shenhav and Zeevi (2020), where a given codon allele 
515 contributes (to either $\mathrm{pN}^{(\text {site) }}$ or $\mathrm{pS}^{(\text {site) }}$ ) an amount that is equal to its observed relative abundance

516 (frequency). To which rate the allele contributes is determined by its synonymity relative to the

517 popular consensus, i.e. the allele most common across all samples. After summing the

518 contributions for each of the 63 codons (excluding the popular consensus), we normalized the

519 resulting values of $\mathrm{pN}^{\text {(site) }}$ and $\mathrm{pS}^{(\text {site) }}$ by the number of nonsynonymous and synonymous sites of

520 the popular consensus, respectively. For example, if the popular consensus is 'ACC' (Thr), there

521 are 9 possible single point mutations, 3 synonymous and 6 nonsynonymous, therefore $\mathrm{pS}^{(\text {site) }}$ will

522 be divided by $3 / 3=1$ and $\mathrm{pN}^{(\text {site })}$ will be divided by $6 / 3=2$. This procedure can be mathematically

523 expressed as

524

$$
p_{N}{ }^{(\text {site })}=\frac{1}{n_{n}} \sum_{c \in C \backslash r} f_{c} N(c, r), \quad p_{S}^{(\text {site })}=\frac{1}{n_{s}} \sum_{c \in C \backslash r} f_{c} S(c, r)
$$

525 Where $C \backslash r$ is the set of all codons excluding the popular consensus $r ; n_{n}$ and $n_{s}$ are the number

526 of nonsynonymous and synonymous sites of $r$, respectively; $f_{c}$ is the frequency of the $c$ th allele;

$527 N(c, r)$ is the indicator function where,

$$
N(c, r)=1 \text { if not synonymous }(c, r) \text { else } 0
$$

529 and $S(c, r)$ is the indicator function where,

$$
S(c, r)=1 \text { if synonymous }(c, r) \text { else } 0 .
$$

531 We implemented this strategy into the program `anvi-gen-variability-profile` as a new flag `--

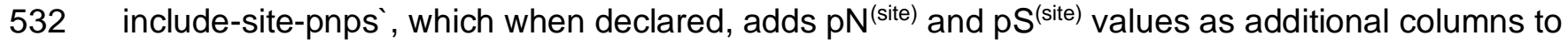
533 the tabular output after calculating them for 3 different choices of the reference codon $r$ : (1) the 534 popular consensus (as used in this paper), (2) the consensus (the allele with the highest 535 frequency), and (3) the codon found in the reference sequence (the sequence used for read 536 recruitment). For efficient computation, this calculation uses the Python package numba (Lam, 
537 Pitrou, and Seibert 2015) for just-in-time compilation. For a dataset with 12,583,626 SCVs, the

538 current implementation computes $\mathrm{pN}^{(\text {(site) }}$ and $\mathrm{pS}^{(\text {site) }}$ terms in less than a minute on a laptop

539 computer.

540 Calculations of polymorphism rates within a group of sites, $\mathbf{p N}^{\text {(group), }} \mathbf{p S}^{\text {(group), and }}$

$541 \mathrm{pN} / \mathbf{p S}^{\text {(group). }}$ We defined groups such that all sites in a group share similar RSA and DTL values.

542 Formally, we defined $\mathrm{pN}^{\text {(group) }}$ and $\mathrm{pS}^{\text {(group) }}$ as

$$
p_{N}^{(\text {group })}=\frac{\sum_{g=1}^{G} \sum_{c \in C \backslash r} f_{c}^{(g)} N\left(c, r^{(g)}\right)}{\sum_{g=1}^{G} n_{n}^{(g)}}, \quad p_{S}^{(\text {group })}=\frac{\sum_{g=1}^{G} \sum_{c \in C \backslash r} f_{c}^{(g)} S\left(c, r^{(g)}\right)}{\sum_{g=1}^{G} n_{S}^{(g)}} .
$$

$544 G$ is the number of sites in the group; $r^{(g)}$ is the popular consensus of the $g$ th site; $f_{c}^{(g)}$ is the

545 frequency of the $c$ th allele at the $g$ th site; $n_{n}{ }^{(g)}$ and $n_{s}{ }^{(g)}$ are the number of nonsynonymous and 546 synonymous sites of $r^{(g)}$, respectively. All other definitions are the same as for $\mathrm{pN}^{(\text {site) }}$ and $\mathrm{pS}^{(\text {(site) }}$.

$547 \mathrm{pN}^{\text {(group) }}$ and $\mathrm{pS}^{\text {(group) }}$ can be expressed in terms of weighted sums of $\mathrm{pN}^{\text {(site) }}$ and $\mathrm{pS}^{\text {(site) }}$, 548 respectively:

$$
p_{N}^{(\text {group })}=\frac{\sum_{g=1}^{G} n_{n}^{(g)} p N^{(g, s i t e)}}{\sum_{g=1}^{G} n_{n}^{(g)}}, \quad p_{S}^{(\text {group })}=\frac{\sum_{g=1}^{G} n_{s}^{(g)} p S^{(g, s i t e)}}{\sum_{g=1}^{G} n_{s}^{(g)}}
$$

550 Finally, $\mathrm{pN} / \mathrm{pS}^{\text {(group) }}$ is defined as

$$
p N / p S^{(\text {group })}=p N^{(\text {group })} / p S^{(\text {group })} .
$$

552 Calculations of polymorphism rates for individual and core genes, $\mathrm{pN}^{\text {(gene) }}, \mathrm{pS}^{\text {(gene) }}$,

$553 \mathrm{pN} / \mathbf{p S}^{\text {(gene) }}$, and $\mathbf{p N} / \mathbf{p S}^{\text {(core) }}$. We calculated rates of polymorphism for genes and the 1a.3.V core

554 genome identically to the calculations of $\mathrm{pN}^{\text {(group) }}, \mathrm{pS}^{\text {(group) }}$, and $\mathrm{pN} / \mathrm{pS}^{\text {(group) }}$. For example, $\mathrm{pN}^{\text {(gene) }}$

555 refers to the ns-polymorphism rate of all sites in a given gene, and $\mathrm{pS}^{(\text {core })}$ refers to the s-

556 polymorphism rate of all sites in the 1a.3.V core genome. 
557 Predicting and processing protein structures. We attempted to predict protein structures for

558 each gene in the HIMB83 genome that belonged to the 1a.3.V core using both AlphaFold (Jumper

559 et al. 2021) and MODELLER (Webb and Sali 2016). To process, store, and access the resulting

560 protein structures we developed a novel program, `anvi-gen-structure-database`, which gives

561 access to all atomic coordinates as well as per-residue statistics such as relative solvent

562 accessibility, secondary structure, and phi \& psi angles calculated using DSSP (Touw et al., 2015;

563 Kabsch and Sander, 1983). For AlphaFold predictions we used a version of the codebase that

564 closely resembles v2.0.1 (this URL gives access to its exact state) and ran predictions using 6

565 GPUs, which took a week on a high-performance computing system. AlphaFold predicted

566 structures for 795 of 799 proteins, and after removing structures with gene-averaged pLDDT

567 scores <80, we were left with 754 structures we deemed 'trustworthy' for downstream analyses.

568 To predict protein structures with MODELLER, we developed a pipeline that, for each gene, (1)

569 searches the Research Collaboratory for Structural Bioinformatics Protein Data Bank (Berman et

570 al. 2000) (RSCB PDB) for homologs using DIAMOND (Buchfink, Xie, and Huson 2015), then

571 downloads tertiary structures for matching entries, and (2) uses these homologs as templates to

572 predict the gene's structure with MODELLER (Webb and Sali 2016). We discarded any proteins

573 if the best template had a percent similarity of $<30 \%$. Unlike more sophisticated homology

574 approaches that make use of multi-domain templates (Källberg et al. 2012), we used single-

575 domain templates which are convenient and are accurate up to several angstroms, yet can lead

576 to physically inaccurate models when the templates' domains match to some, but not all of the

577 sequences' domains. To avoid this, we discarded any templates if the alignment coverage of the

578 protein sequence to the template was $<80 \%$. Applying these filters resulted in 408 structures from

579 the 1a.3.V core, which was further refined by requiring that the root mean squared distance

580 (RMSD) between the predicted structure and the most similar template did not exceed $7.5 \AA$, and

581 that the GA341 model score exceeded 0.95. After applying these constraints, we were left with

582348 structures in the 1a.3.V that we assumed to be 'trustworthy' structures as predicted by 
583 MODELLER. These structures were on average $44.8 \%$ identical to their templates, which is within

584 the sequence similarity regime where template-based homology modeling generally produces the 585 correct overall fold (Rost 1999).

586 Predicting ligand-binding sites. For the 1a.3.V core genes we estimated per-residue binding 587 frequencies for a diverse collection of ligands by using InteracDome, a database that annotates 588 the sites (match states) of Pfam profile hidden Markov models (HMMs) with ligand binding 589 frequencies predicted from experimentally-determined structural data (Kobren and Singh 2019). 590 To associate match state binding frequencies of the profile HMMs to the sites of HIMB83 genes, 591 we applied a protocol similar to that described in Kobren \& Singh.

592 First, we downloaded the Representable-NR Interactions (RNRI) from the InteracDome web server (https://interacdome.princeton.edu/) that "correspond to domain-ligand interactions that 594 had nonredundant instances across three or more distinct PDB structures" (Table S5). Next, we 595 downloaded the profile HMMs for Pfam v31.0 and kept only those 2,375 profiles that belonged to 596 the RNRI dataset. Then, we searched each HIMB83 gene against this set using HMMER's 597 hmmsearch. After the removal of HMM hits that were below the gathering threshold (GA) noise 598 cutoffs defined in Pfam models, 940 of the 1,470 HIMB83 coding genes had at least one domain 599 hit, with a total of 1,770 domain hits from 832 unique profile HMMs. Of these, we removed 177 600 for being too partial (length of the hit divided by the profile HMM length was less than 0.5), and 1 601 hit because the query sequence did not match all the consensus residues for match states in 602 which the information content exceeded 4 (Table S5). We then associated binding frequencies 603 for a collection of ligand types to the HIMB83 genes by parsing alignments of the profile HMMs to 604 the HIMB83 gene amino acid sequences, which are provided in the standard output of 605 hmmsearch. If a given HIMB83 residue aligned to multiple match states, each which had the same 606 ligand type, we attributed the average binding frequency to the HIMB83 residue. We then filtered 607 out binding frequency scores less than 0.5 , yielding 40,219 predicted ligand-residue interactions 
across 11,480 unique sites (Table S5). We considered each of these sites to be ligand-binding

609 sites'.

610 Our study includes two novel programs to automate this procedure and make it accessible to the

611 community. The first, 'anvi-setup-interacdome`, downloads the RNRI and Pfam datasets, and

612 only needs to be run once. The second, 'anvi-run-interacdome', is a multi-threaded program that

613 takes an anvi'o contigs database as input, and runs the remainder of the workflow described for

614 each gene in the database. Predicted binding frequencies are stored internally in the database,

615 which enables a seamless integration with other anvi'o programs to accomplish various tasks,

616 such as the interactive visualization of the binding sites of predicted structures for any given gene

617 with `anvi-display-structure` (see Supplementary Information), or exporting the underlying data as

618 TAB-delimited files with `anvi-export-misc-data`. In the present study, `anvi-run-interacdome`

619 processed the HIMB83 genome in 53 seconds on a laptop computer using a single thread.

620 Calculating relative solvent accessibility (RSA). We calculated RSA for each residue of each

621 predicted structure, where RSA was defined as the accessible surface area (ASA) probed by a

$6221.4 \AA$ radius sphere, divided by the maximum ASA, i.e. the ASA of a Gly-X-Gly tripeptide. RSA

623 values were calculated in the program `anvi-gen-structure-database` using Biopython's DSSP

624 module (Cock et al. 2009).

625 Calculating distance-to-ligand (DTL). DTL was calculated for all sites that belonged to genes

626 with (a) a predicted structure and (b) at least one predicted ligand-binding residue. Ideally, one

627 would calculate DTL as the Euclidean distance of a residue to the predicted ligand, however our

628 predictions did not yield the 3D coordinates of ligands. Instead, we approximated DTL as the

629 Euclidean distance of a residue to the closest ligand-binding residue (see Methods), which lies

630 within a few angstroms of the predicted ligand. Specifically, we defined this distance according to 
631 the sites' side chain center of masses. A consequence of approximating DTL with respect to the

632 closest ligand-binding sites is that by definition, any ligand-binding residue has a DTL of 0 .

633 As discussed in Proteomic trends in purifying selection are explained by RSA and DTL, missed

634 binding sites lead to erroneously high DTL values. We assessed the magnitude of this error

635 source by comparing our distribution of predicted DTL values in the 1a.3.V core to that found in

636 BioLiP, an extensive database of semi-manually curated ligand-protein complexes (Yang, Roy,

637 and Zhang 2013). We found the 1a.3.V DTL distribution had a much higher proportion of values

$638>40 \AA$, suggesting these likely result from incomplete characterization of binding sites (Figure S9).

639 To mitigate the influence of this inevitable error source, we conservatively excluded DTL values

$640>40 \AA$ ( $8.0 \%$ of sites) in all analyses after Figure $2 \mathrm{~b}$.

641 Calculating polymorphism null distributions for RSA and DTL. The null distributions for

642 polymorphism rates with respect to RSA and DTL were calculated by randomly shuffling the RSA

643 and DTL values calculated for each site, yielding distributions one would expect if there was no

644 association between polymorphism rate and RSA. To avoid biases, each null distribution is the

645 average of 10 shuffled datasets.

646 Proportion of polymorphism rate variance explained by RSA and DTL. To calculate the

647 extent that RSA and DTL can explain polymorphism rates, we constructed 3 synonymous models

648 (s-models) and 3 nonsynonymous models (ns-models) (Table S6). s-models fit linear regressions

649 of $\log _{10}\left(\mathrm{pS}^{(\text {site })}\right.$ ) to RSA (s \#1), DTL (s \#2), and both RSA \& DTL (s \#3). Similarly, ns-models fit

650 linear regressions of $\log _{10}\left(\mathrm{pN}^{(\text {site) }}\right)$ to RSA (ns \#1), DTL (ns \#2), and both RSA \& DTL (ns \#3).

651 Additionally, each model included the gene and sample of the corresponding polymorphism as

652 independent variables, in order to account for gene-to-gene and sample-to-sample differences.

653 Polymorphism rates were log-transformed because it helped linearize the data, yielding better

654 models. The data used to fit each model included all codon positions across all samples in each 
655 gene that had a predicted protein structure and at least 1 predicted ligand-binding residue. After

656 excluding monomorphic sites $\left(\mathrm{pN}^{(\text {site })}=0\right.$ for ns-models, $\mathrm{pS}^{(\text {site })}=0$ for s-models), this yielded

$6575,838,445$ data points for s-models and 3,850,182 for ns-models. While every protein has RSA

658 values that span the domain $[0,1]$, protein size creates dramatic gene-to-gene differences in

659 observed DTL values. We accounted for this by standardizing DTL values on a per-gene basis,

660 which improved variance explained by DTL. The variance explained by RSA, DTL, sample, and

661 gene was determined by performing an ANOVA on each model and partitioning the sum of 662 squares (Table S6).

663 Calculating transcript abundance (TA). Since proper transcription level metrics such as 664 molecules per cell are incalculable from metatranscriptomic data, we estimated the transcript 665 abundance (TA) to be

$$
T A=\frac{C^{(M T)}}{D^{(M T)}} / \frac{C^{(M G)}}{D^{(M G)}}
$$

667 Where $C^{(M T)}$ is the coverage of the gene in the metatranscriptome, $D^{(M T)}$ is the sequencing depth 668 (total number of reads) of the metatranscriptome, $C^{(M G)}$ is the coverage of the gene in the 669 metagenome, and $D^{(M T)}$ is the sequencing depth (total number of reads) of the metagenome.

670 This means, for example, that a gene with a metatranscriptomic relative abundance $10 \%$ of its 671 metagenomic relative abundance would have a TA of 0.10 .

672 Definition of codon rarity. We defined the rarity of a codon in the following way. First, we 673 calculated the unnormalized codon rarity for each codon $c$, which we defined as

$$
R_{c}^{\prime}=\left(1-f_{c}\right)
$$

675 where $f_{c}$ is the frequency that a codon was observed in the HIMB83 genome sequence. Then, 676 we normalized the values such that the codons with the lowest and highest values get rarity scores 677 of 0 and 1 , respectively: 


$$
R_{c}=\left(\left(R^{\prime}{ }_{c}-\min \left(R^{\prime}\right)\right) /\left(\max \left(R^{\prime}\right)-\min \left(R^{\prime}\right)\right)\right.
$$

679 where $\min \left(R^{\prime}\right)$ and $\max \left(R^{\prime}\right)$ correspond to the smallest and largest unnormalized rarity scores.

680 We utilized this definition to calculate codon rarity at polymorphic sites by weighting each codon's 681 rarity by the frequency that the codon was observed in the short reads mapping to that position. 682 For example, a polymorphic site with a coverage of 200 , where 50 reads resolve to GCC $\left(R_{G C C}=\right.$ $6830.97)$ and 150 resolve to $\operatorname{GCT}\left(R_{G C C}=0.75\right)$ would get a rarity score of $50 / 200 \times 0.97+$ $684150 / 200 \times 0.75=0.81$. Extending this to multiple sites, we take the codon rarity of an entire 685 sample to be the average rarity across all codon sites (polymorphic or not).

686 Statistical data analysis and visualization. We used R v3.5.1 ( $R$ Development Core Team 687 2011) for the analysis of numerical data reported from anvi'o. For data visualization we used 688 ggplot2 (Ginestet 2011) library in R and anvi'o, and finalized images for publication using Inkscape 689 v1.1 (https://inkscape.org/). 


\section{Acknowledgements}

We thank Stephen Giovannoni (https://microbiology.oregonstate.edu/dr-stephen-giovannoni), Shilpa Nadimpalli Kobren (http://shilpakobren.com/), Michael K. Yu (@michaelkuyu), Tobin Sosnick (http://sosnick.uchicago.edu/), as well as the members of our laboratory (https://merenlab.org/people/) for helpful discussions. EK acknowledges support from the Natural Sciences and Engineering Research Council of Canada. This work was supported by Alfred P. Sloan Foundation Fellowship in Ocean Sciences to AME, and by a Simons Foundation grant (\#687269) to AME.

\section{Author contributions}

EK and AME conceptualized the study and interpreted findings. EK curated data, developed software tools, and performed primary analyses. OCE, and AME contributed software. EK and AME wrote the paper. SEM, KK, and ADW helped with data analyses and interpretation. MSP and TP helped with project management and funding acquisition. AME supervised the project. All authors commented on the drafts of the study. All authors read and approved the final manuscript.

\section{Ethics declarations}

\section{Competing interests}

Authors have no competing interests to declare. 


\section{Supplementary Figures}

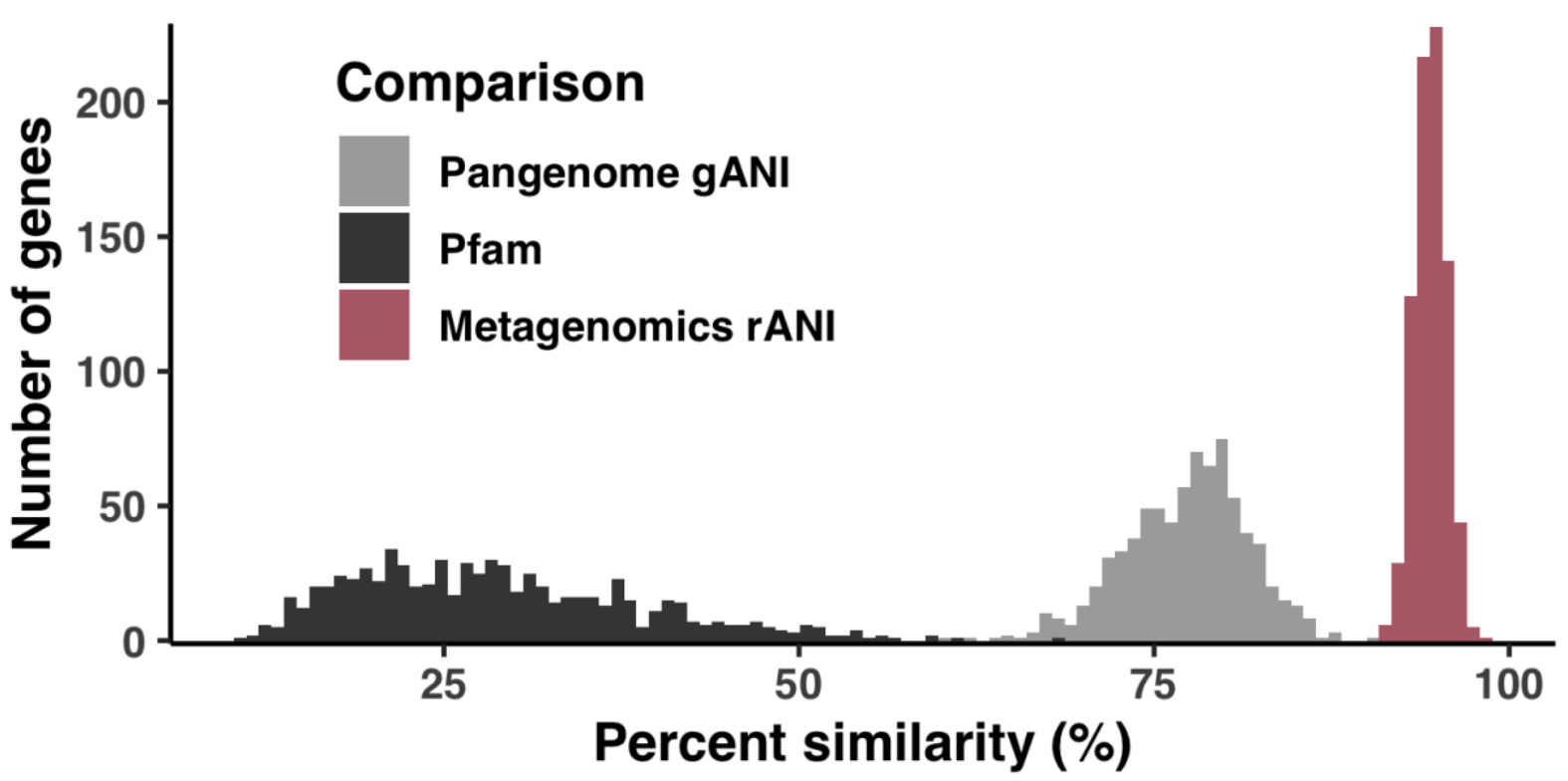

Figure S1. Regimes of sequence similarity probed by metagenomics, SAR11 cultured genomes, and protein

692 families. Empirical distributions of gene-level percent similarity for HIMB83 compared with recruited metagenomic 693 reads (pink), homologous SAR11 genomes (blue), and homologous Pfams (orange). For calculation details, see 694 Supplementary Information. 

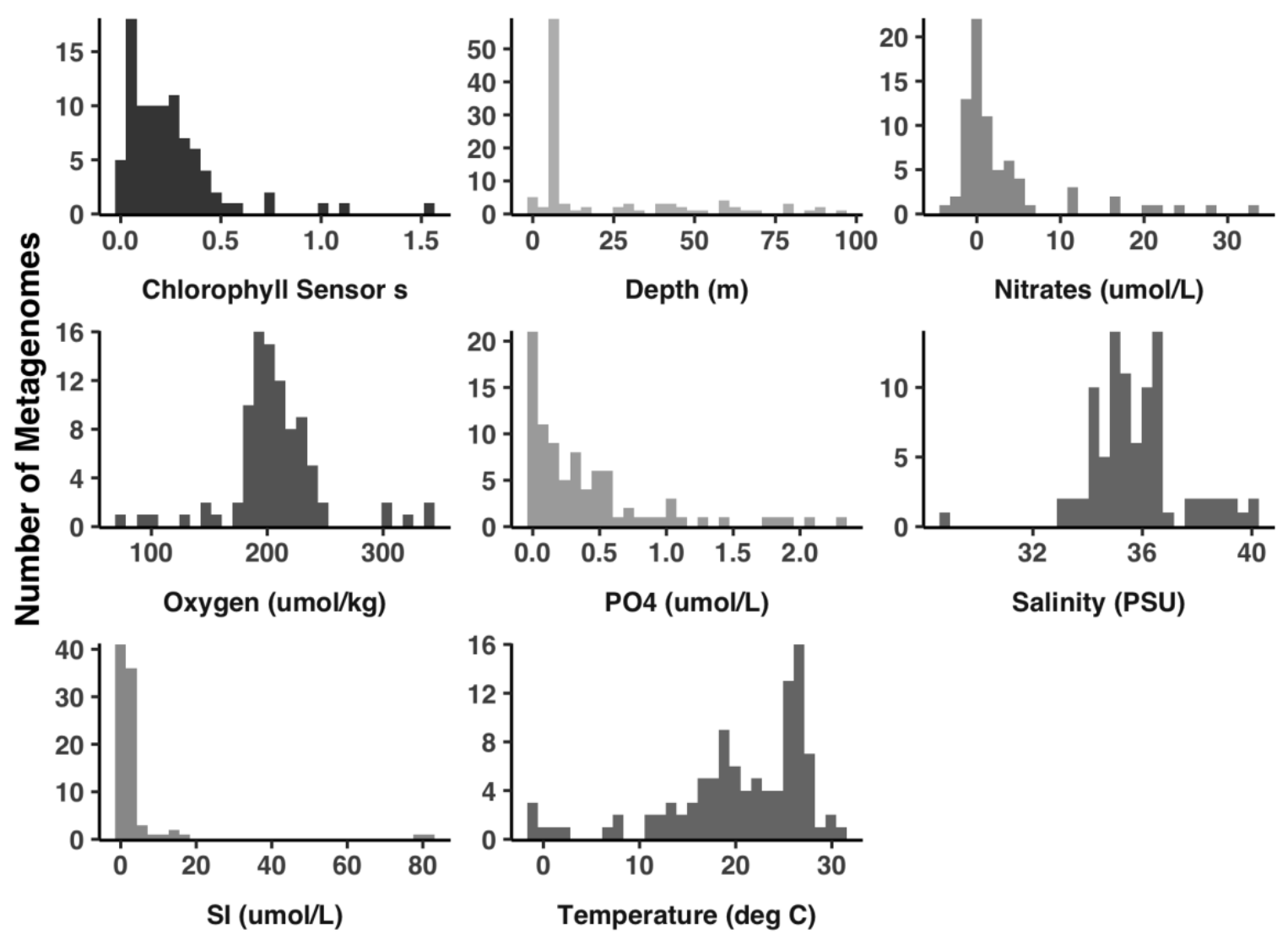

696 Figure S2. Different environments exhibit substantial variation in their environmental parameters. Each subplot

697 shows how the 74 selected metagenomes distribute according to various environmental variables measured by the 698 TARA ocean metagenome project. 


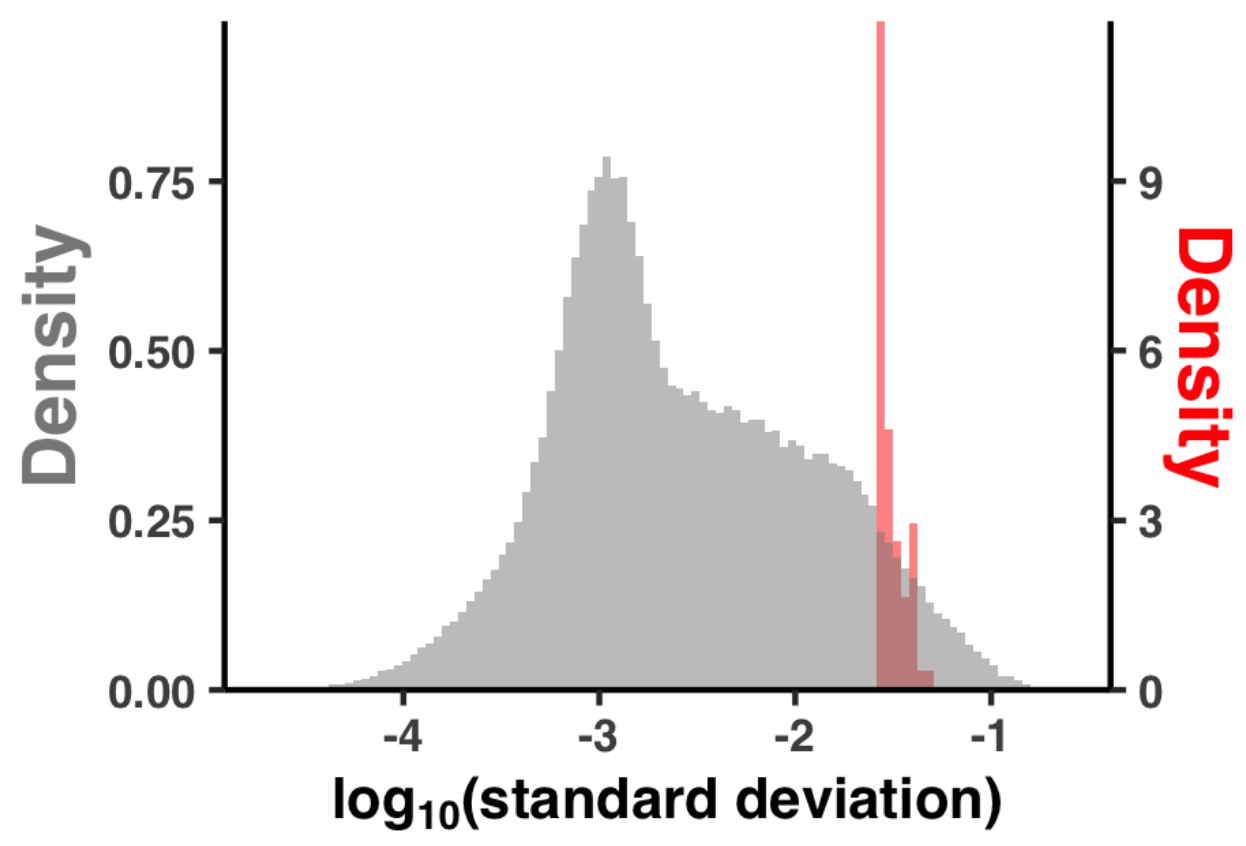

701 Figure S3. $\mathrm{pN}^{\text {(site) }}$ varies more significantly between sites in a given sample than between samples for a given

702 site. The $\mathrm{x}$-axis is the log-transformed standard deviation of either a sample's $\mathrm{pN}^{\text {(site) }}$ values observed over many sites 703 (orange), or a site's $\mathrm{pN}^{\text {(site) }}$ values observed over the 74 samples (gray). 
A

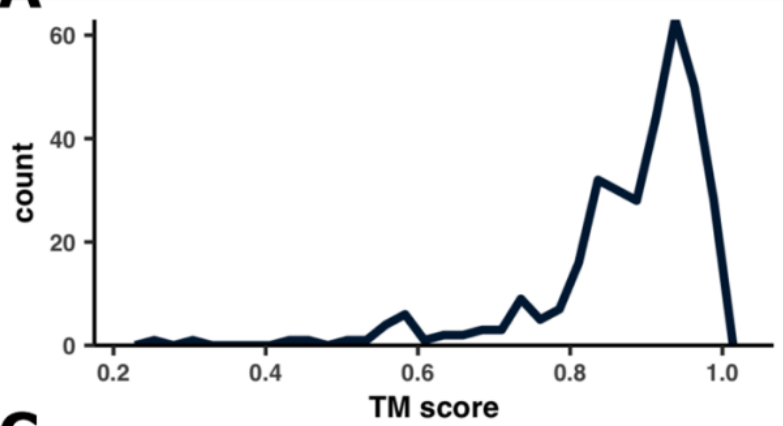

C

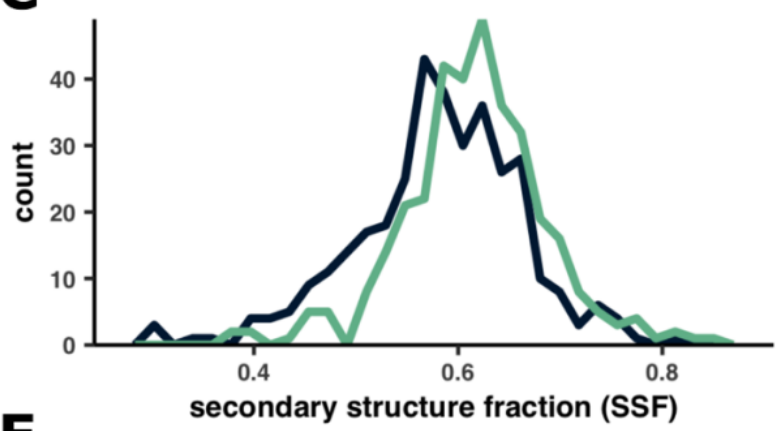

$\mathbf{E}$

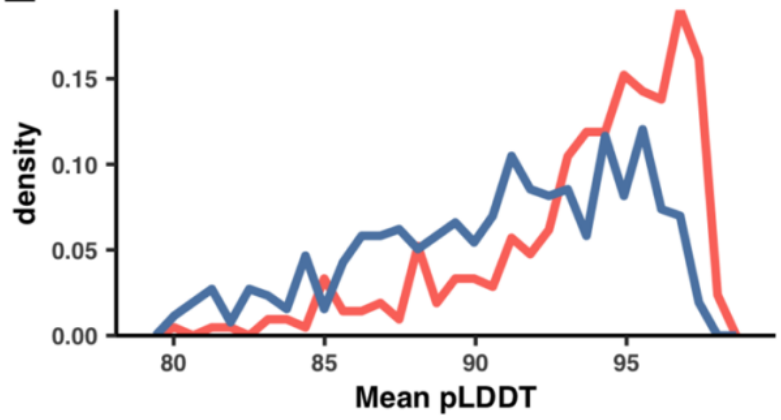

B

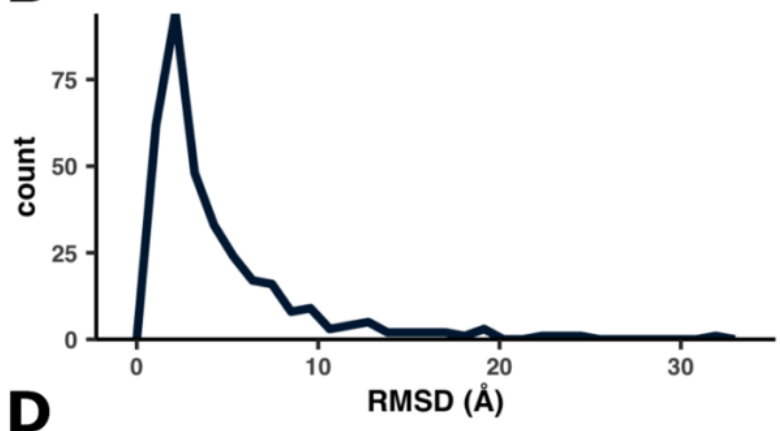

D

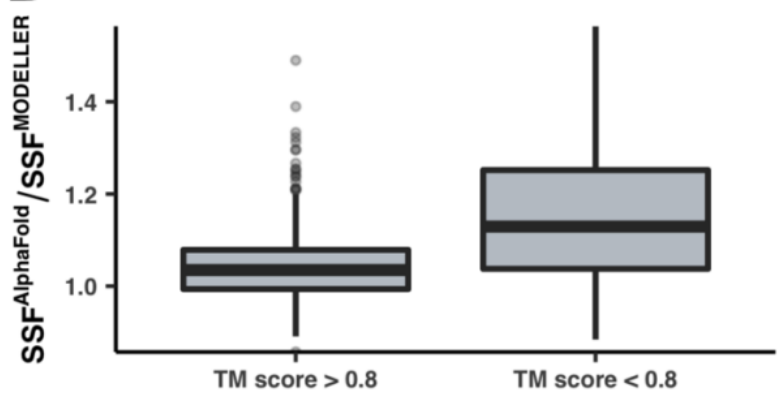

$\mathbf{F}$

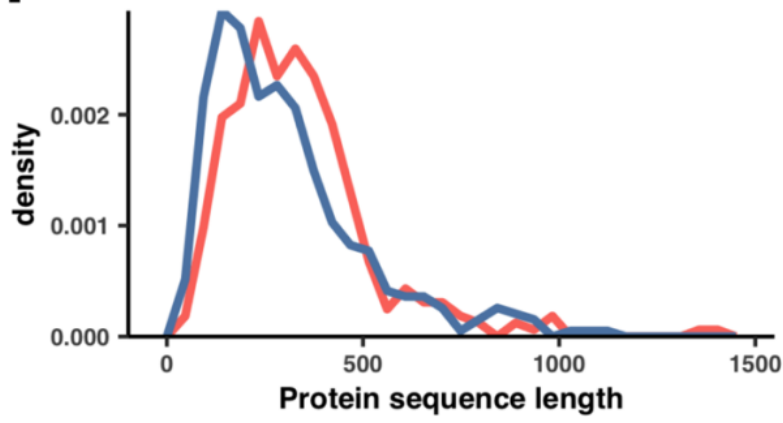

Figure S4. Comparisons between structures predicted by AlphaFold and MODELLER. (A-B) Distributions of TM scores and RMSD between structures predicted by both MODELLER and AlphaFold. (C) Distribution of secondary structure fractions, between MODELLER (black) and AlphaFold (green). Secondary structure fraction was defined for each gene as the fraction of sites that DSSP predicted as part of an alpha helix or beta strand. (D) Comparison of secondary structure fractions between MODELLER and AlphaFold for two TM score groups. The $y$-axis is the secondary structure fraction of AlphaFold divided by the secondary structure fraction of MODELLER. The two groups

711 were defined as having TM scores above or below 0.8 , where the $>0.8$ group corresponded to the 291 best alignments

712 (left) and the $<0.8$ group corresponded to the 48 worst alignments. (E-F) Distributions describing the mean pLDDT and 713 protein sequence length of AlphaFold structures that either (1) had analog MODELLER structures (red) or (2) did not 714 (blue). 
bioRxiv preprint doi: https://doi.org/10.1101/2022.03.02.482602; this version posted March 2, 2022. The copyright holder for this preprint (which was not certified by peer review) is the author/funder, who has granted bioRxiv a license to display the preprint in perpetuity. It is made available under aCC-BY-NC-ND 4.0 International license.
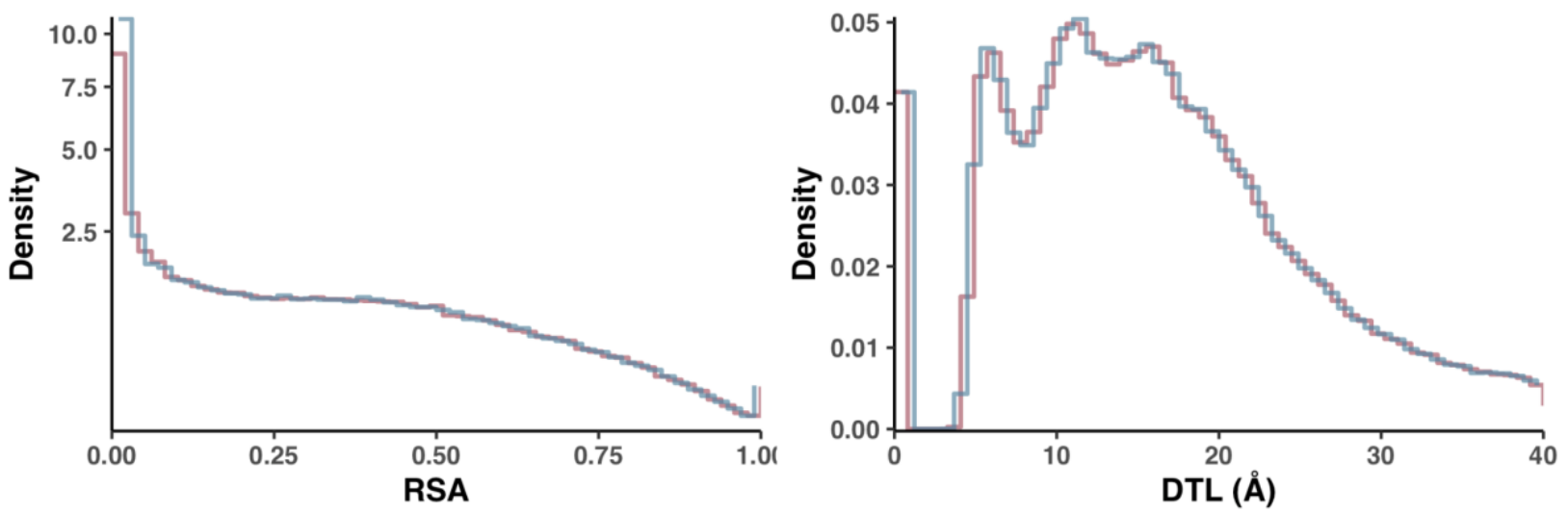

716 Figure S5. Comparison of null distributions for $\mathrm{pN}^{\text {(site) }}$ and $\mathrm{pS}^{\text {(site) }}$ for RSA and DTL. Each distribution was

717 calculated by averaging 10 independent, randomly shuffled datasets of either $\mathrm{pN}^{\text {(site) }}$ (red line) or $\mathrm{pS}^{\text {(site) }}$ (blue line). To

718 better visualize differences between the null distributions, the blue lines depicting the $\mathrm{pS}$ (site) distributions were shifted

719 right by half of a bin's width. 

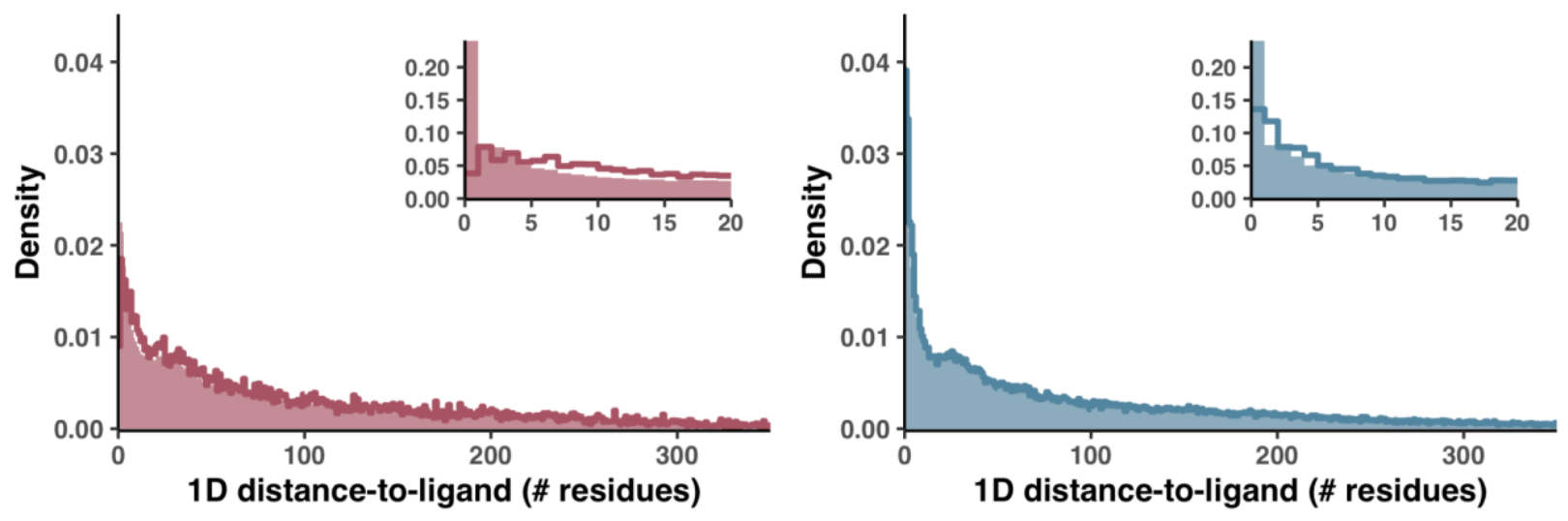

721 Figure S6. Functional constraint is less resolved when using a sequence-distance metric of DTL. pN(site) (left

722 panel) and $\mathrm{pS}$ (site) (right panel) distributions with respect to 1D DTL, which we defined as the number of sites in a

723 protein's sequence that separate a given site from a predicted ligand-binding site. Lines represent the observed

724 distributions, and filled regions represent the null distributions, calculated via the shuffling procedure described in Figure

725 2. Insets show the same data zoomed into the $1 \mathrm{D}$ DTL range $[0,20]$. 
bioRxiv preprint doi: https://doi.org/10.1101/2022.03.02 482602; this version posted March 2, 2022. The copyright holder for this preprint (which was not certified by peer review) is the author/funder, who has granted bioRxiv a license to display the preprint in perpetuity. It is made available under aCC-BY-NC-ND 4.0 International license.
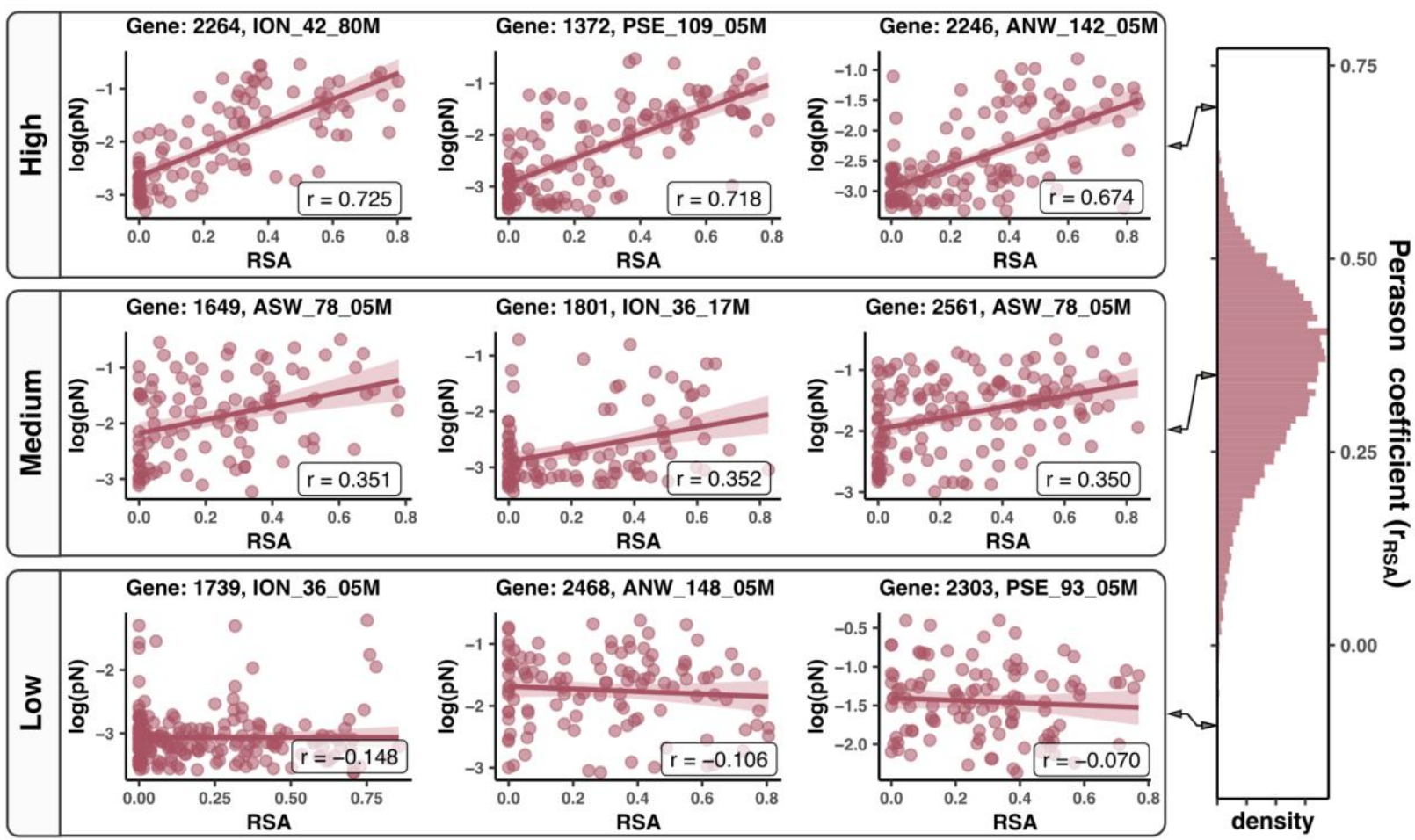

Figure S7. Select gene-sample pairs illustrate the diversity with which $\mathrm{pN}^{(\text {site) }}$ associates with RSA. Scatterplots for handpicked gene-sample pairs are shown from three regimes of model quality: high (top), mid (middle), and low (bottom). The right panel shows the distribution of Pearson coefficients, and the bin that each example was taken from is highlighted in pink. Each scatter plot is a gene-sample pair, each datapoint is a residue, the x-axis is the RSA of the residue, and the $y$-axis is the observed $\log _{10}\left(\mathrm{pN}^{(\text {site })}\right)$. Lines of best fit are shown in red, with $95 \%$ confidence intervals visualized translucently. The Pearson coefficients of each fit are labeled on the scatterplot. 
bioRxiv preprint doi: https://doi.org/10.1101/2022.03.02.482602; this version posted March 2, 2022. The copyright holder for this preprint (which was not certified by peer review) is the author/funder, who has granted bioRxiv a license to display the preprint in perpetuity. It is made available under aCC-BY-NC-ND 4.0 International license.
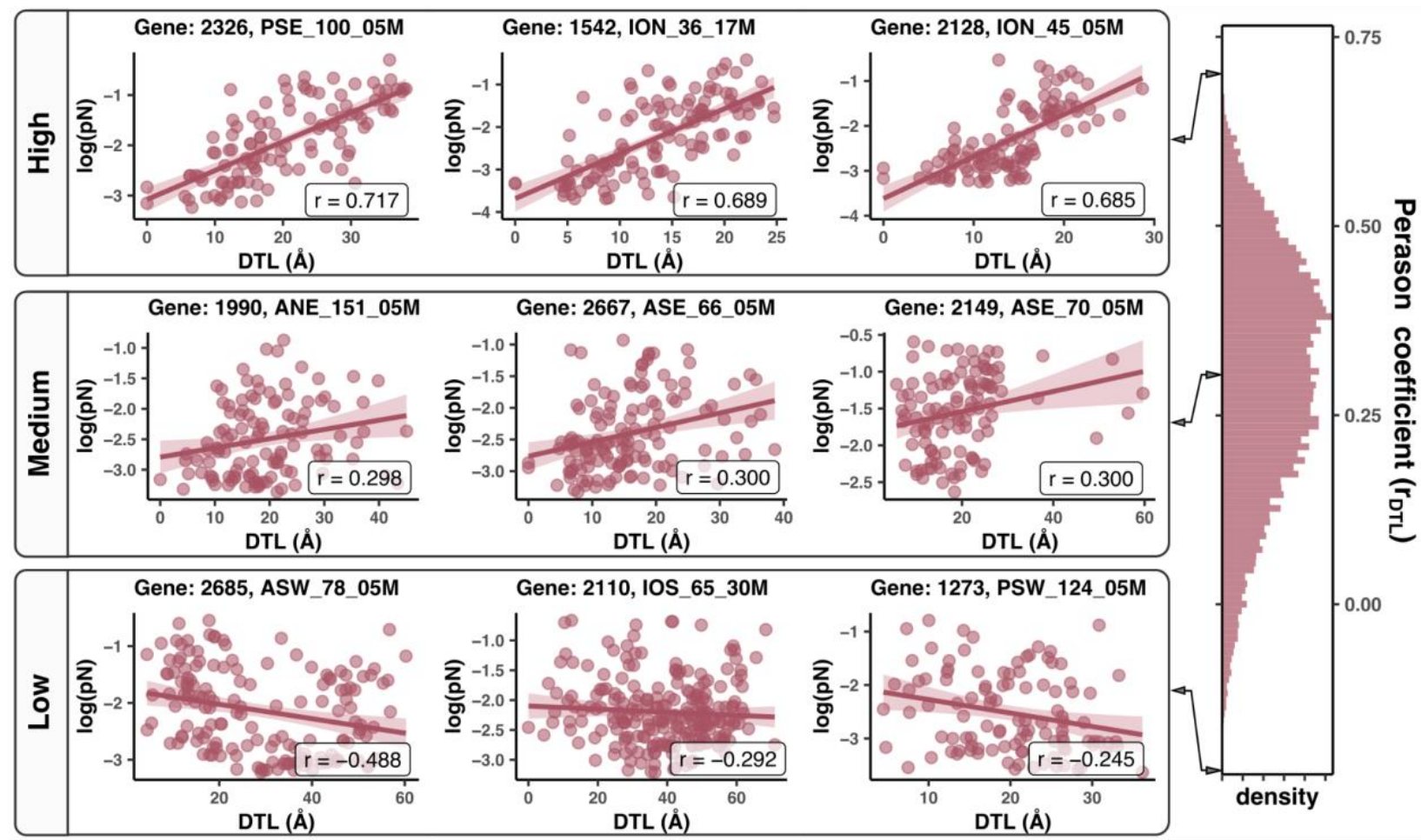

Figure S8. Select gene-sample pairs illustrate the diversity with which $\mathrm{pN}^{(\text {(site) }}$ associates with DTL.

735 Scatterplots for handpicked gene-sample pairs are shown from three regimes of model quality: high (top), mid

736 (middle), and low (bottom). The right panel shows the distribution of Pearson coefficients, and the bin that each example was taken from is highlighted in pink. Each scatter plot is a gene-sample pair, each datapoint is a residue, the $x$-axis is the DTL of the residue, and the $y$-axis is the observed $\log _{10}(\mathrm{pN}($ site) $)$. Lines of best fit are shown in red, with $95 \%$ confidence intervals visualized translucently. The Pearson coefficients of each fit are labeled on the scatterplot. 


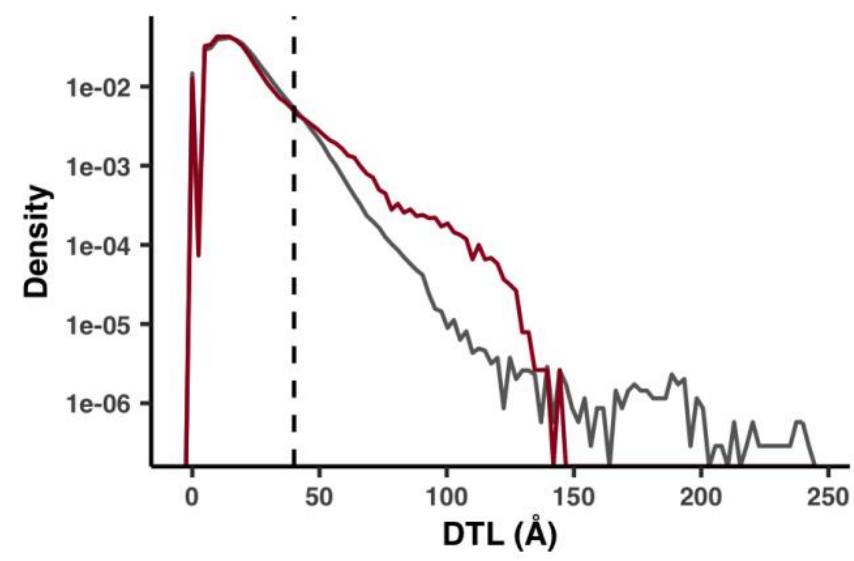

742 Figure 59. Incomplete ligand characterization leads to erroneously high DTL values. A comparison of DTL

743 distributions (semi-log axis) for the 1a.3.V and the BioLiP database. The 1a.3.V core distribution (red) was calculated

744 from all sites in the subset of genes with both a predicted structure and at least one predicted ligand-binding residue.

745 The BioLiP distribution (gray) was calculated from the sites of 5,000 structures in the BioLiP database. For the 1a.3.V

746 core, DTL was calculated as the distance to the closest predicted ligand-binding residue. For BioLiP, it was calculated

747 as the distance to the closest annotated ligand-binding residue. For both methods, distance was calculated between

748 the sites' side chain center of masses. The dashed line marks the $40 \AA$ cutoff we used for all analyses besides Figure

$7492 \mathrm{~b}$, which excludes $8.0 \%$ of the total sites. 


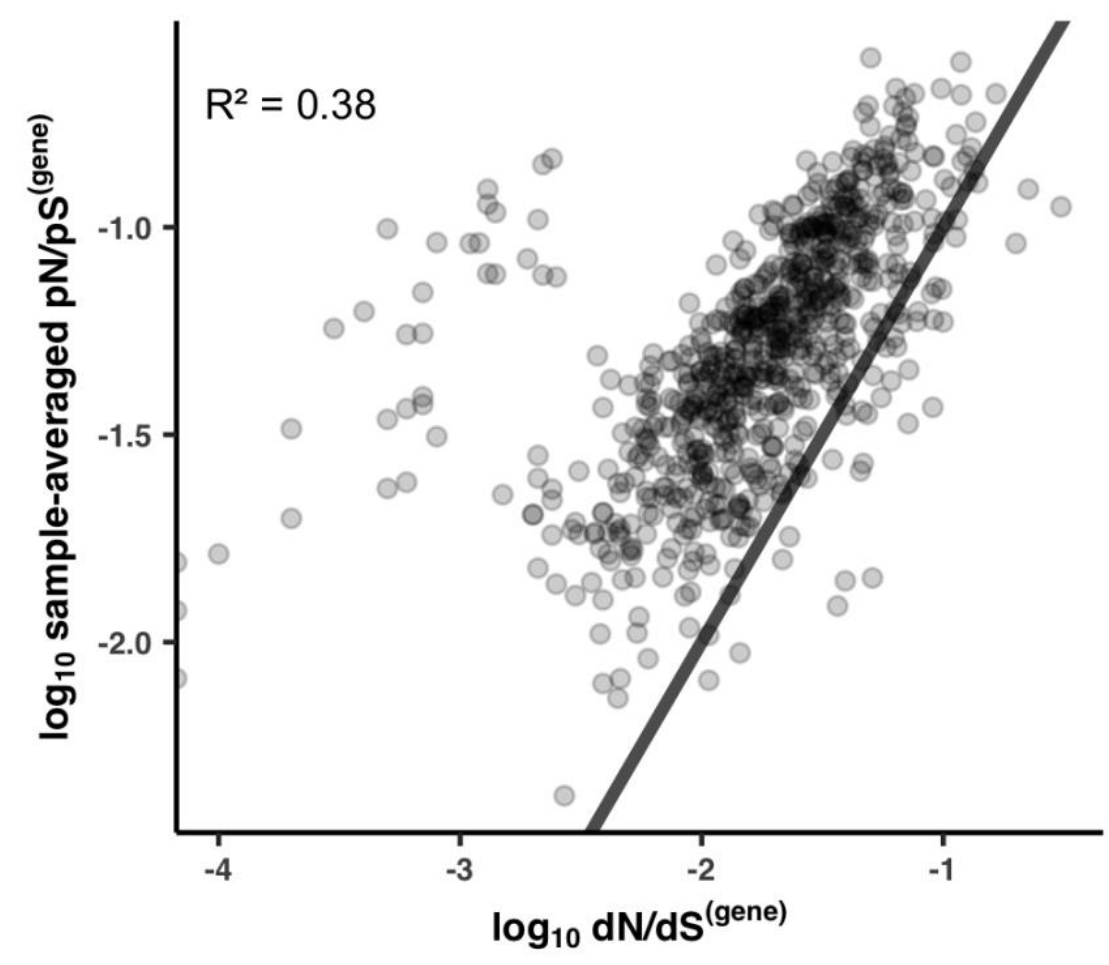

752 Figure S10. Sample-averaged $\mathrm{pN} / \mathrm{pS}^{(\text {gene) }}$ values correlate with $\mathrm{dN} / \mathrm{dS}^{(g e n e)}$ values between HIMB83 and

753 HIMB122. The $x$ - and $y$-axes are the log-transformed dN/dS ${ }^{\text {(gene) }}$ and sample-averaged $\mathrm{pN} / \mathrm{pS}^{\text {(gene) }}$ values (respectively)

754 for the 743 genes that (1) belonged to the 1a.3.V core and (2) had HIMB122 homologs. The black line is the equation

$755 y=x$, meaning that genes above this line maintain sample-averaged $p N / p S^{(\text {gene) }}$ values that exceed dN/dS(gene). The $R^{2}$

756 is for a linear regression of the log-transformed variables. 


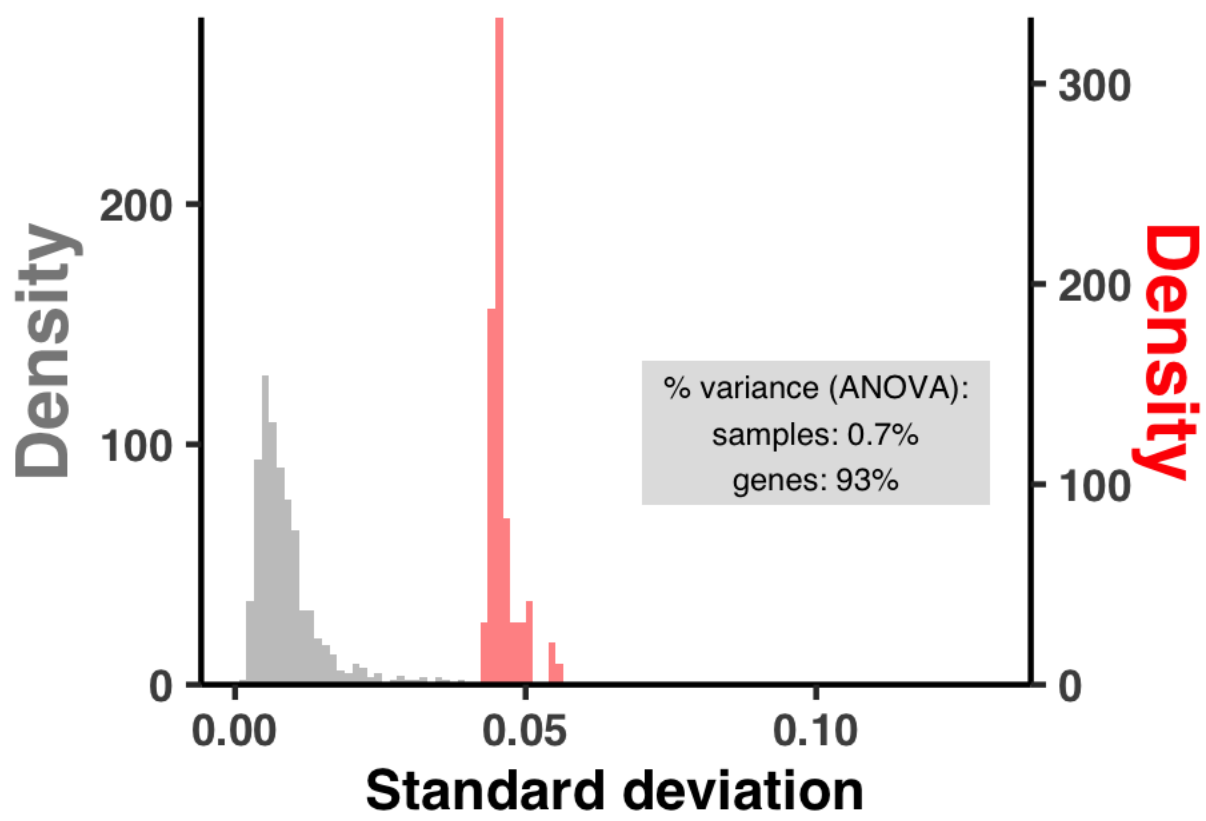

758 Figure S11. pN/pS (gene) varies more significantly between genes in a given sample than between samples for a

759 given gene. The $x$-axis is the standard deviation of either a sample's $\mathrm{pN} / \mathrm{pS}^{\text {(gene) }}$ values observed over genes (orange),

760 or a gene's $\mathrm{pN} / \mathrm{pS}^{(\text {gene) }}$ values observed over the 74 samples (gray). The gray box denotes the amount of variance

761 explained by genes and samples in an ANOVA from the linear model pN/pS(gene) $~$ gene + sample. 

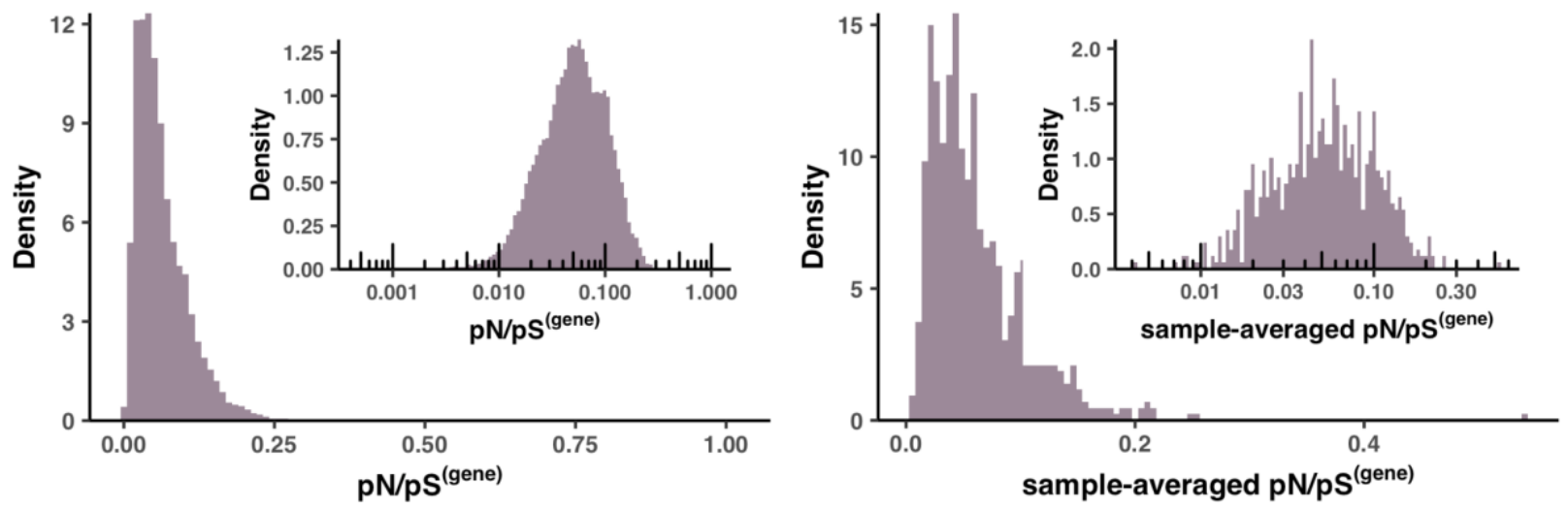

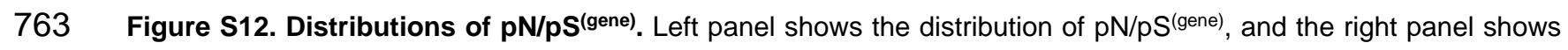

764 the distribution of sample-averaged $\mathrm{pN} / \mathrm{pS}^{\text {(gene). }}$. Insets show the same distributions with a $\log _{10}$-transformed $\mathrm{x}$-axis. 
bioRxiv preprint doi: https://doi.org/10.1101/2022.03.02 482602; this version posted March 2, 2022. The copyright holder for this preprint (which was not certified by peer review) is the author/funder, who has granted bioRxiv a license to display the preprint in perpetuity. It is made available under aCC-BY-NC-ND 4.0 International license.

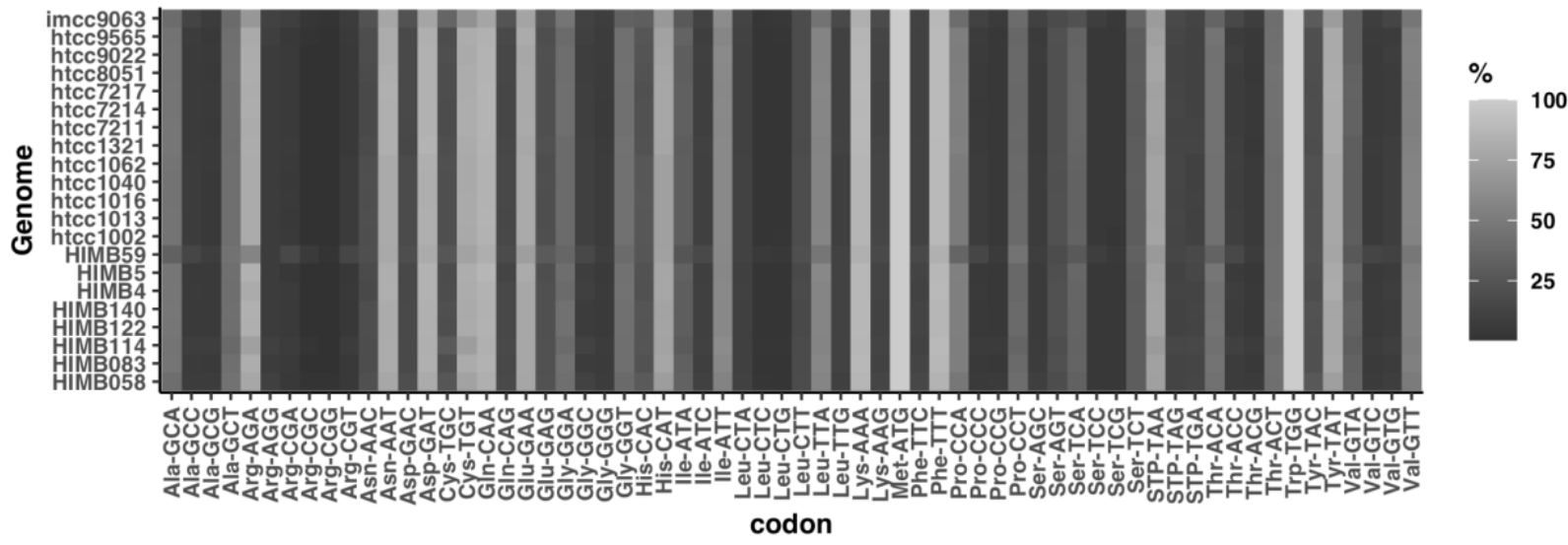

Figure S13. Codon usage of HIMB83 and 20 other genomes in the SAR11 clade. 
bioRxiv preprint doi: https://doi.org/10.1101/2022.03.02.482602; this version posted March 2, 2022. The copyright holder for this preprint (which was not certified by peer review) is the author/funder, who has granted bioRxiv a license to display the preprint in perpetuity. It is made available under aCC-BY-NC-ND 4.0 International license.
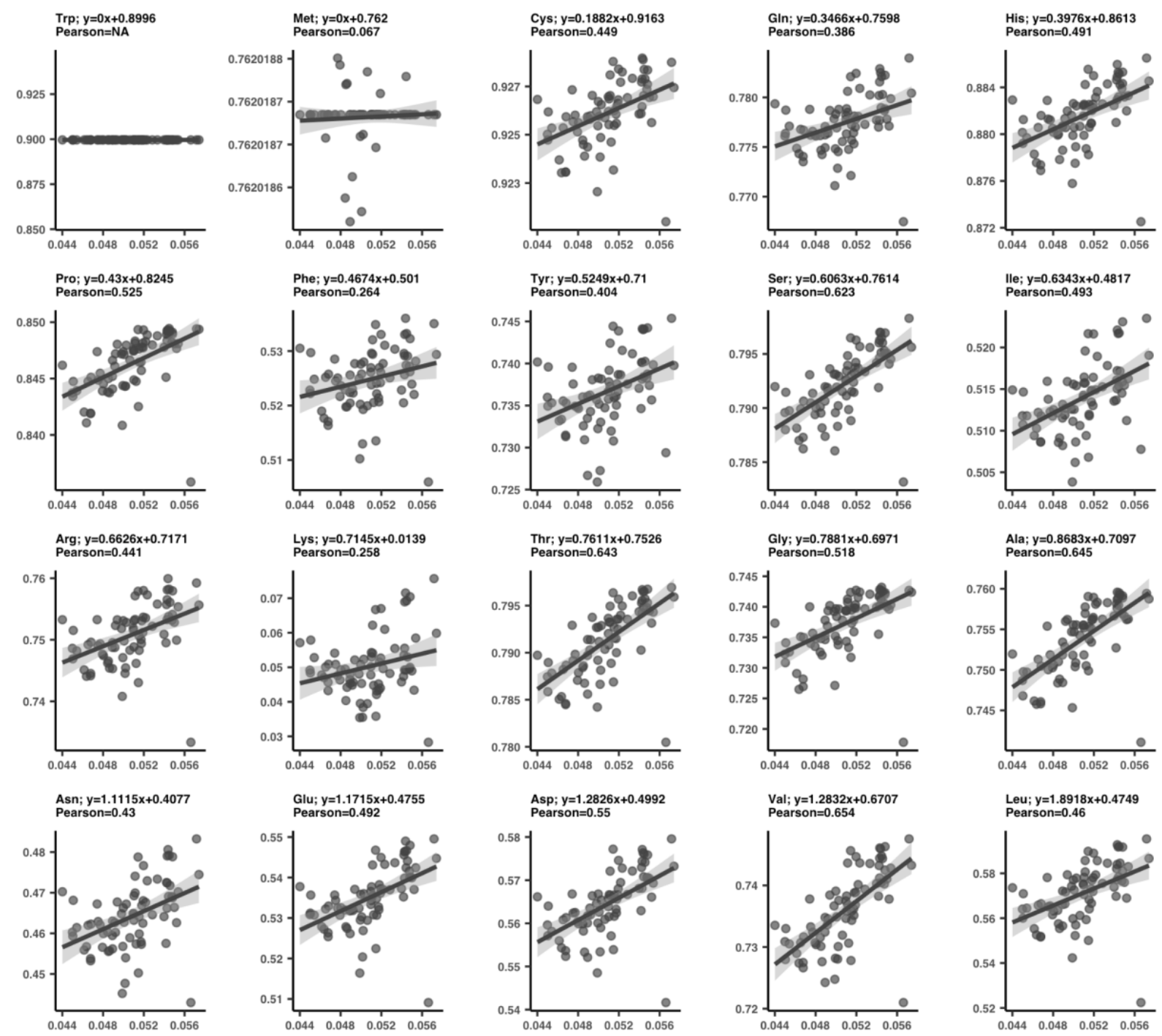

Figure S14. Codon rarity measured for each amino acid reveals varied response to selection strength, with core genome, and is shared between all plots. For a given plot, the $y$-axis was determined by first subsetting the polymorphism data to only include synonymous sites (in this instance we define synonymous as exhibiting $\mathrm{pN}^{\text {(site) }}<$

773 0.0005) that corresponded to the given amino acid. Using lysine as an example, this led to on average 21,127 sites per

774 sample. For each amino acid in each sample, we then calculated the overall codon rarity ( $y$-axis) by averaging codon

775 rarities across all included positions. A line of best fit (gray line) with 95\% confidence intervals (light gray) is shown for 776 each plot, with equation and Pearson correlation coefficient shown above. 


\section{Supplementary Tables}

777 Table S1. Read recruitment and coverage statistics of the 21 SAR11 genomes. (A-D) Genome-wide statistics for each

778 genome in each metatranscriptomic and metagenomic sample. (A) is the mean coverage, (B) is the mean coverage,

779 excluding nucleotide coverage values outside the interquartile range (IQR), (C) is the detection, and (D) is the

780 percentage of reads mapping to a genome (sums to 100 for a given sample) (E) The mean coverage of each HIMB083

781 gene in each metatranscriptomic and metagenomic sample.

782 Table S2. Average percent similarity of recruited reads by HIMB083 for each (A) gene-sample pair, (B) gene 783 (marginalized over samples), and (C) sample (marginalized over genes).

784 Table S3. Mean per-site polymorphism rates ( $\mathrm{pN}^{(\text {site) }}$ and $\left.\mathrm{pS}^{(\text {site) }}\right)$ of $\mathrm{HIMB083}(\mathbf{A})$ over all sites, genes, and samples, 785 as well as (B) for each gene-sample pair (C) each gene (marginalized over samples), and (D) each sample 786 (marginalized over genes).

787 Table S4. Methodological comparisons between AlphaFold and MODELLER structures. (A) Key metrics for AlphaFold-

788 and MODELLER-predicted structures and their alignments. (B) PDB structures used as templates for MODELLER 789 predictions. (C) Per-residue pLDDT scores for AlphaFold-predicted structures. (D) Gene-averaged pLDDT scores for 790 AlphaFold-predicted structures. (E-F) Genes with AlphaFold and MODELLER structures, respectively, that we 791 determined to be of sufficiently high quality.

792 Table S5. Summary of ligand-binding residue predictions with InteracDome. (A) All predicted ligand-binding sites, the 793 predicted ligand, and the predicted ligand binding score. (B) Characterization of each HMM domain hit. (C) Each match 794 state from the Pfam profile HMMs that contributed to each predicted ligand-binding residue of HIMB083. 


\begin{tabular}{|c|c|c|c|c|c|c|}
\hline & & \multicolumn{5}{|c|}{ Sum of squares (\% of total) } \\
\hline Name & Model & RSA & DTL & Gene & Sample & Residuals \\
\hline s \#1 & $\log _{10}\left(\mathrm{pS}^{(\text {site })}\right) \sim \mathrm{RSA}+$ gene + sample & 0.12 & - & 4.05 & 1.01 & 94.83 \\
\hline ns \#1 & $\log _{10}(\mathrm{pN}($ site) $) \sim \mathrm{RSA}+$ gene + sample & 11.83 & - & 10.61 & 6.71 & 70.85 \\
\hline s \#2 & $\log _{10}\left(p S^{(\text {site })}\right) \sim D T L+$ gene + sample & - & 0.30 & 4.07 & 1.00 & 94.63 \\
\hline ns \#2 & $\log _{10}\left(p S^{(\text {site })}\right) \sim \mathrm{DTL}+$ gene + sample & - & 6.89 & 12.08 & 7.10 & 74.39 \\
\hline $\mathrm{s} \# 3$ & $\begin{array}{c}\log _{10}\left(\mathrm{pS}^{(\text {site })}\right) \underset{\text { sample }}{\sim} \mathrm{RSA}+\mathrm{DTL}+\text { gene }+ \\
\text { sate }\end{array}$ & 0.03 & 0.30 & 4.05 & 1.00 & 94.62 \\
\hline ns \#3 & $\begin{array}{c}\log _{10}\left(\mathrm{pS}^{(\text {site })}\right) \\
\sim \mathrm{RSA}+\mathrm{DTL}+\text { gene + } \\
\text { sample }\end{array}$ & 7.23 & 6.89 & 10.83 & 6.42 & 68.62 \\
\hline
\end{tabular}

Table S6. Summary of models used for estimating the explanatory power of RSA and DTL on polymorphism rates (see 798 Methods).

Table S7. Summary statistics for the polymorphism models of gene-sample pairs.

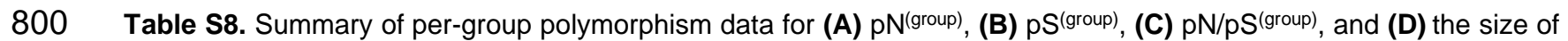
801 each group.

802 Table S9. Summary of per-gene polymorphism data for (A) pN/pS (gene), (B) sample-averaged pN/pS(gene), (C) pN(gene),

803 (D) $\mathrm{pS}^{\text {(gene) }}$ and (E) the number of potential synonymous and nonsynonymous point mutations of each gene.

804 Table S10. Correlations of $\mathrm{pN} / \mathrm{pS}^{\text {(gene) }}$ for each 1a.3.V core gene with respect to the measured environmental 805 parameters: nitrates, chlorophyll, temperature, salinity, phosphate, silicon, depth, and oxygen.

806 Table S11. Codon metrics, including anti-codon, encoded amino acid, frequency and rarity in genome, and frequency 807 and rarity compared to synonymous codons.

808 Table S12. Comparison between dN/dS between HIMB83 and HIMB122 homologs and sample-averaged pN/pS(gene) 809 of 1a.3.V genes.

810 Table S13. Per sample and gene measures of transcript abundance (TA) and related quantities.

811 Table S14. Bootstrap estimates of Pearson correlation coefficients and p-values from Figure SI6. 


\section{References}

Acinas, Silvia G., Vanja Klepac-Ceraj, Dana E. Hunt, Chanathip Pharino, Ivica Ceraj, Daniel L. Distel, and Martin F. Polz. 2004. "Fine-Scale Phylogenetic Architecture of a Complex Bacterial Community." Nature 430 (6999): $551-$ 54.

Agashe, Deepa, N. Cecilia Martinez-Gomez, D. Allan Drummond, and Christopher J. Marx. 2013. "Good Codons, Bad Transcript: Large Reductions in Gene Expression and Fitness Arising from Synonymous Mutations in a Key Enzyme." Molecular Biology and Evolution 30 (3): 549-60.

Allen, Eric E., Gene W. Tyson, Rachel J. Whitaker, John C. Detter, Paul M. Richardson, and Jillian F. Banfield. 2007. "Genome Dynamics in a Natural Archaeal Population." Proceedings of the National Academy of Sciences of the United States of America 104 (6): 1883-88.

Almeida, Alexandre, Stephen Nayfach, Miguel Boland, Francesco Strozzi, Martin Beracochea, Zhou Jason Shi, Katherine S. Pollard, et al. 2021. "A Unified Catalog of 204,938 Reference Genomes from the Human Gut Microbiome." Nature Biotechnology 39 (1): 105-14.

Anderson, Rika E., Julie Reveillaud, Emily Reddington, Tom O. Delmont, A. Murat Eren, Jill M. McDermott, Jeff S. Seewald, and Julie A. Huber. 2017. "Genomic Variation in Microbial Populations Inhabiting the Marine Subseafloor at Deep-Sea Hydrothermal Vents." Nature Communications 8 (1): 1114.

Anfinsen, C. B. 1973. "Principles That Govern the Folding of Protein Chains." Science 181 (4096): 223-30.

Bendall, Matthew L., Sarah Lr Stevens, Leong-Keat Chan, Stephanie Malfatti, Patrick Schwientek, Julien Tremblay, Wendy Schackwitz, et al. 2016. "Genome-Wide Selective Sweeps and Gene-Specific Sweeps in Natural Bacterial Populations." The ISME Journal 10 (7): 1589-1601.

Berman, H. M., J. Westbrook, Z. Feng, G. Gilliland, T. N. Bhat, H. Weissig, I. N. Shindyalov, and P. E. Bourne. 2000. "The Protein Data Bank." Nucleic Acids Research 28 (1): 235-42.

Bernard, Stéphanie M., and Dimah Z. Habash. 2009. "The Importance of Cytosolic Glutamine Synthetase in Nitrogen Assimilation and Recycling." The New Phytologist 182 (3): 608-20.

Bristow, Laura A., Wiebke Mohr, Soeren Ahmerkamp, and Marcel M. M. Kuypers. 2017. "Nutrients That Limit Growth in the Ocean." Current Biology: CB 27 (11): R474-78.

Buchfink, Benjamin, Chao Xie, and Daniel H. Huson. 2015. "Fast and Sensitive Protein Alignment Using DIAMOND." Nature Methods. https://doi.org/10.1038/nmeth.3176.

Burke, Molly K., Joseph P. Dunham, Parvin Shahrestani, Kevin R. Thornton, Michael R. Rose, and Anthony D. Long. 2010. "Genome-Wide Analysis of a Long-Term Evolution Experiment with Drosophila." Nature 467 (7315): 58790.

Chen, K., and F. H. Arnold. 1993. "Tuning the Activity of an Enzyme for Unusual Environments: Sequential Random Mutagenesis of Subtilisin E for Catalysis in Dimethylformamide." Proceedings of the National Academy of Sciences of the United States of America 90 (12): 5618-22.

Chen, Lin-Xing, Karthik Anantharaman, Alon Shaiber, A. Murat Eren, and Jillian F. Banfield. 2020. "Accurate and Complete Genomes from Metagenomes." Genome Research 30 (3): 315-33.

Cock, Peter J. A., Tiago Antao, Jeffrey T. Chang, Brad A. Chapman, Cymon J. Cox, Andrew Dalke, Iddo Friedberg, et al. 2009. "Biopython: Freely Available Python Tools for Computational Molecular Biology and Bioinformatics." Bioinformatics 25 (11): 1422-23.

Conwill, Arolyn, Anne C. Kuan, Ravalika Damerla, Alexandra J. Poret, Jacob S. Baker, A. Delphine Tripp, Eric J. Alm, and Tami D. Lieberman. 2022. "Anatomy Promotes Neutral Coexistence of Strains in the Human Skin Microbiome." Cell Host \& Microbe, January. https://doi.org/10.1016/j.chom.2021.12.007.

Costea, Paul Igor, Robin Munch, Luis Pedro Coelho, Lucas Paoli, Shinichi Sunagawa, and Peer Bork. 2017. "metaSNV: A Tool for Metagenomic Strain Level Analysis." PloS One 12 (7): e0182392.

Curtis, Thomas P., Ian M. Head, Mary Lunn, Stephen Woodcock, Patrick D. Schloss, and William T. Sloan. 2006. "What Is the Extent of Prokaryotic Diversity?" Philosophical Transactions of the Royal Society of London. Series B, Biological Sciences 361 (1475): 2023-37.

Curtis, T. P., and W. T. Sloan. 2005. "Microbiology. Exploring Microbial Diversity--a Vast below." Science.

Dean, Antony M., Claudia Neuhauser, Elise Grenier, and G. Brian Golding. 2002. "The Pattern of Amino Acid Replacements in Alpha/beta-Barrels." Molecular Biology and Evolution 19 (11): 1846-64.

Delmont, Tom O., Evan Kiefl, Ozsel Kilinc, Ozcan C. Esen, Ismail Uysal, Michael S. Rappé, Steven Giovannoni, and A. Murat Eren. 2019. "Single-Amino Acid Variants Reveal Evolutionary Processes That Shape the Biogeography of a Global SAR11 Subclade." eLife 8 (September). https://doi.org/10.7554/eLife.46497.

Denef, Vincent J. 2019. "Peering into the Genetic Makeup of Natural Microbial Populations Using Metagenomics." In Population Genomics: Microorganisms, edited by Martin F. Polz and Om P. Rajora, 49-75. Cham: Springer International Publishing.

Drummond, D. Allan, and Claus O. Wilke. 2008. "Mistranslation-Induced Protein Misfolding as a Dominant Constraint on Coding-Sequence Evolution." Cell 134 (2): 341-52. 
Echave, Julian, Stephanie J. Spielman, and Claus O. Wilke. 2016. "Causes of Evolutionary Rate Variation among Protein Sites." Nature Reviews. Genetics 17 (2): 109-21.

Eddy, Sean R. 2011. "Accelerated Profile HMM Searches." PLoS Computational Biology 7 (10): e1002195.

El-Gebali, Sara, Jaina Mistry, Alex Bateman, Sean R. Eddy, Aurélien Luciani, Simon C. Potter, Matloob Qureshi, et al. 2019. "The Pfam Protein Families Database in 2019." Nucleic Acids Research 47 (D1): D427-32.

Eren, A. Murat, Özcan C. Esen, Christopher Quince, Joseph H. Vineis, Hilary G. Morrison, Mitchell L. Sogin, and Tom O. Delmont. 2015. "Anvi'o: An Advanced Analysis and Visualization Platform for 'omics Data." PeerJ 3 (October): e1319.

Eren, A. Murat, Evan Kiefl, Alon Shaiber, Iva Veseli, Samuel E. Miller, Matthew S. Schechter, Isaac Fink, et al. 2021. "Community-Led, Integrated, Reproducible Multi-Omics with Anvi'o." Nature Microbiology 6 (1): 3-6.

Garud, Nandita R., Benjamin H. Good, Oskar Hallatschek, and Katherine S. Pollard. 2019. "Evolutionary Dynamics of Bacteria in the Gut Microbiome within and across Hosts." PLoS Biology 17 (1): e3000102.

Garud, Nandita R., and Katherine S. Pollard. 2020. "Population Genetics in the Human Microbiome." Trends in Genetics: TIG 36 (1): 53-67.

Ginestet, Cedric. 2011. "ggplot2: Elegant Graphics for Data Analysis: Book Reviews." Journal of the Royal Statistical Society. Series A, $174(1): 245-46$.

Giovannoni, Stephen J. 2017. "SAR11 Bacteria: The Most Abundant Plankton in the Oceans." Annual Review of Marine Science 9 (January): 231-55.

Golding, G. B., and A. M. Dean. 1998. "The Structural Basis of Molecular Adaptation." Molecular Biology and Evolution 15 (4): 355-69.

Good, Benjamin H., Michael J. McDonald, Jeffrey E. Barrick, Richard E. Lenski, and Michael M. Desai. 2017. "The Dynamics of Molecular Evolution over 60,000 Generations." Nature 551 (7678): 45-50.

Goodsell and, David S., and Arthur J. Olson. 2003. "Structural Symmetry and Protein Function," November. https://doi.org/10.1146/annurev.biophys.29.1.105.

Harms, Michael J., and Joseph W. Thornton. 2013. "Evolutionary Biochemistry: Revealing the Historical and Physical Causes of Protein Properties." Nature Reviews. Genetics 14 (8): 559-71.

Haro-Moreno, Jose M., Francisco Rodriguez-Valera, Riccardo Rosselli, Francisco Martinez-Hernandez, Juan J. Roda-Garcia, Monica Lluesma Gomez, Oscar Fornas, Manuel Martinez-Garcia, and Mario López-Pérez. 2020. "Ecogenomics of the SAR11 Clade." Environmental Microbiology 22 (5): 1748-63.

Hug, Laura A., Brett J. Baker, Karthik Anantharaman, Christopher T. Brown, Alexander J. Probst, Cindy J. Castelle, Cristina N. Butterfield, et al. 2016. "A New View of the Tree of Life." Nature Microbiology 1 (April): 16048.

Hyatt, Doug, Gwo-Liang Chen, Philip F. Locascio, Miriam L. Land, Frank W. Larimer, and Loren J. Hauser. 2010. "Prodigal: Prokaryotic Gene Recognition and Translation Initiation Site Identification." BMC Bioinformatics 11 (March): 119.

Jumper, John, Richard Evans, Alexander Pritzel, Tim Green, Michael Figurnov, Olaf Ronneberger, Kathryn Tunyasuvunakool, et al. 2021. "Highly Accurate Protein Structure Prediction with AlphaFold." Nature 596 (7873): 583-89.

Källberg, Morten, Haipeng Wang, Sheng Wang, Jian Peng, Zhiyong Wang, Hui Lu, and Jinbo Xu. 2012. "TemplateBased Protein Structure Modeling Using the RaptorX Web Server." Nature Protocols 7 (8): 1511-22.

Kobren, Shilpa Nadimpalli, and Mona Singh. 2019. "Systematic Domain-Based Aggregation of Protein Structures Highlights DNA-, RNA- and Other Ligand-Binding Positions." Nucleic Acids Research 47 (2): 582-93.

Komar, Anton A. 2009. "A Pause for Thought along the Co-Translational Folding Pathway." Trends in Biochemical Sciences 34 (1): 16-24.

Köster, Johannes, and Sven Rahmann. 2012. "Snakemake--a Scalable Bioinformatics Workflow Engine." Bioinformatics 28 (19): 2520-22.

Kuhlman, Brian, and Philip Bradley. 2019. "Advances in Protein Structure Prediction and Design." Nature Reviews. Molecular Cell Biology 20 (11): 681-97.

Lam, Siu Kwan, Antoine Pitrou, and Stanley Seibert. 2015. "Numba: A LLVM-Based Python JIT Compiler." In Proceedings of the Second Workshop on the LLVM Compiler Infrastructure in HPC, 1-6. LLVM '15 7. New York, NY, USA: Association for Computing Machinery.

Langmead, Ben, and Steven L. Salzberg. 2012. "Fast Gapped-Read Alignment with Bowtie 2." Nature Methods 9 (4): 357-59.

Lenski, Richard E., Michael R. Rose, Suzanne C. Simpson, and Scott C. Tadler. 1991. "Long-Term Experimental Evolution in Escherichia Coli. I. Adaptation and Divergence During 2,000 Generations." The American Naturalist 138 (6): 1315-41.

Li, Heng, Bob Handsaker, Alec Wysoker, Tim Fennell, Jue Ruan, Nils Homer, Gabor Marth, Goncalo Abecasis, Richard Durbin, and 1000 Genome Project Data Processing Subgroup. 2009. "The Sequence Alignment/Map Format and SAMtools." Bioinformatics 25 (16): 2078-79.

Mes, Ted H. M. 2008. "Microbial Diversity--Insights from Population Genetics." Environmental Microbiology 10 (1): 251-64.

Morris, Robert M., Michael S. Rappé, Stephanie A. Connon, Kevin L. Vergin, William A. Siebold, Craig A. Carlson, and Stephen J. Giovannoni. 2002. "SAR11 Clade Dominates Ocean Surface Bacterioplankton Communities." 
Nature 420 (6917): 806-10.

Nayfach, Stephen, Beltran Rodriguez-Mueller, Nandita Garud, and Katherine S. Pollard. 2016. "An Integrated Metagenomics Pipeline for Strain Profiling Reveals Novel Patterns of Bacterial Transmission and Biogeography." Genome Research 26 (11): 1612-25.

Ochman, Howard. 2003. "Neutral Mutations and Neutral Substitutions in Bacterial Genomes." Molecular Biology and Evolution 20 (12): 2091-96.

Olm, Matthew R., Alexander Crits-Christoph, Keith Bouma-Gregson, Brian A. Firek, Michael J. Morowitz, and Jillian F. Banfield. 2021. "inStrain Profiles Population Microdiversity from Metagenomic Data and Sensitively Detects Shared Microbial Strains." Nature Biotechnology 39 (6): 727-36.

Olsen, G. J., D. J. Lane, S. J. Giovannoni, N. R. Pace, and D. A. Stahl. 1986. "Microbial Ecology and Evolution: A Ribosomal RNA Approach." Annual Review of Microbiology 40: 337-65.

Pachiadaki, Maria G., Julia M. Brown, Joseph Brown, Oliver Bezuidt, Paul M. Berube, Steven J. Biller, Nicole J. Poulton, et al. 2019. "Charting the Complexity of the Marine Microbiome through Single-Cell Genomics." Cell 179 (7): 1623-35.e11.

Pál, C., B. Papp, and L. D. Hurst. 2001. "Highly Expressed Genes in Yeast Evolve Slowly." Genetics 158 (2): $927-31$.

Paoli, Lucas, Hans-Joachim Ruscheweyh, Clarissa C. Forneris, Satria Kautsar, Quentin Clayssen, Guillem Salazar, Alessio Milanese, et al. 2021. "Uncharted Biosynthetic Potential of the Ocean Microbiome." bioRxiv. https://doi.org/10.1101/2021.03.24.436479.

Plotkin, Joshua B., and Grzegorz Kudla. 2011. "Synonymous but Not the Same: The Causes and Consequences of Codon Bias." Nature Reviews. Genetics 12 (1): 32-42.

Quince, Christopher, Alan W. Walker, Jared T. Simpson, Nicholas J. Loman, and Nicola Segata. 2017. "Shotgun Metagenomics, from Sampling to Analysis." Nature Biotechnology 35 (9): 833-44.

R Development Core Team, R. 2011. R: A Language and Environment for Statistical Computing. https://doi.org/10.1007/978-3-540-74686-7.

Rost, B. 1999. "Twilight Zone of Protein Sequence Alignments." Protein Engineering 12 (2): 85-94.

Salazar, Guillem, Lucas Paoli, Adriana Alberti, Jaime Huerta-Cepas, Hans-Joachim Ruscheweyh, Miguelangel Cuenca, Christopher M. Field, et al. 2019. "Gene Expression Changes and Community Turnover Differentially Shape the Global Ocean Metatranscriptome." Cell 179 (5): 1068-83.e21.

Schloissnig, Siegfried, Manimozhiyan Arumugam, Shinichi Sunagawa, Makedonka Mitreva, Julien Tap, Ana Zhu, Alison Waller, et al. 2013. "Genomic Variation Landscape of the Human Gut Microbiome." Nature 493 (7430): 45-50.

Shaiber, Alon, Amy D. Willis, Tom O. Delmont, Simon Roux, Lin-Xing Chen, Abigail C. Schmid, Mahmoud Yousef, et al. 2020. "Functional and Genetic Markers of Niche Partitioning among Enigmatic Members of the Human Oral Microbiome." Genome Biology 21 (1): 292.

Shenhav, Liat, and David Zeevi. 2020. "Resource Conservation Manifests in the Genetic Code." Science 370 (6517): 683-87.

Sikosek, Tobias, and Hue Sun Chan. 2014. "Biophysics of Protein Evolution and Evolutionary Protein Biophysics." Journal of the Royal Society, Interface / the Royal Society 11 (100): 20140419.

Siltberg-Liberles, Jessica, Johan A. Grahnen, and David A. Liberles. 2011. "The Evolution of Protein Structures and Structural Ensembles under Functional Constraint." Genes 2 (4): 748-62.

Simmons, Sheri L., Genevieve Dibartolo, Vincent J. Denef, Daniela S. Aliaga Goltsman, Michael P. Thelen, and Jillian F. Banfield. 2008. "Population Genomic Analysis of Strain Variation in Leptospirillum Group II Bacteria Involved in Acid Mine Drainage Formation." PLoS Biology 6 (7): e177.

Smith Daniel P., Thrash J. Cameron, Nicora Carrie D., Lipton Mary S., Burnum-Johnson Kristin E., Carini Paul, Smith Richard D., Giovannoni Stephen J., and McFall-Ngai Margaret J. n.d. "Proteomic and Transcriptomic Analyses of 'Candidatus Pelagibacter Ubique' Describe the First PII-Independent Response to Nitrogen Limitation in a FreeLiving Alphaproteobacterium." mBio 4 (6): e00133-12.

Sogin, Mitchell L., Hilary G. Morrison, Julie A. Huber, David Mark Welch, Susan M. Huse, Phillip R. Neal, Jesus M. Arrieta, and Gerhard J. Herndl. 2006. "Microbial Diversity in the Deep Sea and the Underexplored 'Rare Biosphere." Proceedings of the National Academy of Sciences of the United States of America 103 (32): 1211520.

Sørensen, M. A., C. G. Kurland, and S. Pedersen. 1989. "Codon Usage Determines Translation Rate in Escherichia Coli." Journal of Molecular Biology 207 (2): 365-77.

Sunagawa, Shinichi, Luis Pedro Coelho, Samuel Chaffron, Jens Roat Kultima, Karine Labadie, Guillem Salazar, Bardya Djahanschiri, et al. 2015. "Ocean Plankton. Structure and Function of the Global Ocean Microbiome." Science 348 (6237): 1261359.

Sunyaev, S., W. Lathe 3rd, and P. Bork. 2001. "Integration of Genome Data and Protein Structures: Prediction of Protein Folds, Protein Interactions and 'Molecular Phenotypes' of Single Nucleotide Polymorphisms." Current Opinion in Structural Biology 11 (1): 125-30.

Tatusov, Roman L., Natalie D. Fedorova, John D. Jackson, Aviva R. Jacobs, Boris Kiryutin, Eugene V. Koonin, Dmitri M. Krylov, et al. 2003. "The COG Database: An Updated Version Includes Eukaryotes." BMC Bioinformatics 4 (September): 41. 
Van Rossum, Thea, Pamela Ferretti, Oleksandr M. Maistrenko, and Peer Bork. 2020. "Diversity within Species: Interpreting Strains in Microbiomes." Nature Reviews. Microbiology 18 (9): 491-506.

Walsh, Ian M., Micayla A. Bowman, Iker F. Soto Santarriaga, Anabel Rodriguez, and Patricia L. Clark. 2020. "Synonymous Codon Substitutions Perturb Cotranslational Protein Folding in Vivo and Impair Cell Fitness." Proceedings of the National Academy of Sciences of the United States of America 117 (7): 3528-34.

Webb, Benjamin, and Andrej Sali. 2016. "Comparative Protein Structure Modeling Using MODELLER." Current Protocols in Bioinformatics / Editoral Board, Andreas D. Baxevanis ... [et Al.] 54 (June): 5.6.1-5.6.37.

Whitaker, Rachel J., and Jillian F. Banfield. 2006. "Population Genomics in Natural Microbial Communities." Trends in Ecology \& Evolution 21 (9): 508-16.

Wilke, Claus O. 2012. "Bringing Molecules Back into Molecular Evolution." PLoS Computational Biology 8 (6): e1002572.

Woyke, Tanja, Devin F. R. Doud, and Frederik Schulz. 2017. "The Trajectory of Microbial Single-Cell Sequencing." Nature Methods 14 (11): 1045-54.

Yang, Jianyi, Ambrish Roy, and Yang Zhang. 2013. "BioLiP: A Semi-Manually Curated Database for Biologically Relevant Ligand-Protein Interactions." Nucleic Acids Research 41 (Database issue): D1096-1103.

Zhao, Shijie, Tami D. Lieberman, Mathilde Poyet, Kathryn M. Kauffman, Sean M. Gibbons, Mathieu Groussin, Ramnik J. Xavier, and Eric J. Alm. 2019. "Adaptive Evolution within Gut Microbiomes of Healthy People." Cell Host \& Microbe 25 (5): 656-67.e8. 